\title{
Genome-wide Identification of Gramineae Histone Modification Genes and their Potential Roles in Regulating Wheat and Maize Growth and Stress Responses
}

\section{Liwei Zheng}

Zhengzhou university

Shengjie Ma

Zhengzhou University

Dandan Shen

Zhengzhou University

Hong Fu

Zhengzhou University

\section{Yue Wang}

Zhengzhou University

Ying Liu

Zhengzhou University

Kamran Shah

Zhengzhou University

Caipeng Yue

Zhengzhou University

Jinyong Huang ( $\square$ jinyhuang@zzu.edu.cn )

Zhengzhou University

Research article

Keywords: Histone modification, wheat and maize, growth and development, stress

Posted Date: November 10th, 2020

DOl: https://doi.org/10.21203/rs.3.rs-101516/v1

License: (c) (1) This work is licensed under a Creative Commons Attribution 4.0 International License.

Read Full License 
Version of Record: A version of this preprint was published at BMC Plant Biology on November 20th, 2021. See the published version at https://doi.org/10.1186/s12870-021-03332-8. 


\section{Abstract}

Background: In pants, histone modification (HM) participates in various developmental and defenses processes. Gramineae plants were important crop species worldwide. However, little information on them is in gramineae species.

Results: In six gramineaes, $245 \mathrm{TaHMs}, 72 \mathrm{HvHMs}, 84 \mathrm{SbHMs}, 93 \mathrm{SvHMs}, 90 \mathrm{SiHMs}$ and $90 \mathrm{ZmHMs}$ were respectively identified. Their detailed information, including chromosome locations, conserved domains, phylogenetic trees, synteny, promoter elements and gene structures, were identified. Among these $H M S$, most of motifs were conservative, while unique ones were also identified. Gene and genome duplications may result in the evolution and expansion of $H M s$ in wheat. The number of gene pairs between rice and each gramineae was much greater than that between Arabidopsis and each gramineae, which indicated dicotyledons sharing common ancestors. Moreover, all identified HMs gene pairs may undergo purifying selection according to their $\mathrm{Ka} / \mathrm{Ks}$ values. Expression profiles of TaHMs in developing wheat grain, responding to brassinosteroid, brassinazole as well as activated charcoal were investigated in published transcriptome data, and transcription models of $Z m H M s$ in maize development seeds and after gibberellin treatment were also identified. In addition, heat, drought, salt, insect feeding, nitrogen and cadmium stresses influenced many TaHMs, and drought altered several $\mathrm{ZmHMs}$ expression. These findings indicated their important functions in plant growth and stress adaptions.

Conclusion: In conclusion, a comprehensive analysis of six gramineae $H M$ gene families was completed; TaHMs were likely to participate in grain development, brassinosteroid- as well as brassinazole-mediated root growth, activated charcoal-mediated root and leaf growth, and biotic and abiotic adaptions; ZmHMs take part in seed development, gibberellin-mediated leaf growth, and drought adaption.

\section{Background}

In plants, histone modification (HM) is an important epigenetic type which can activate or silence gene expression. HMs genes play essential functions in a series of growth and development processes and stress responses, such as carotenoid biosynthesis, floral organ development and fungal pathogens resistance, directly or indirectly. HM process depend on four kinds of proteins, including histone methyltransferases (HMTs), histone demethylases (HDMs), histone acetylases (HATs), and histone deacetylases (HDACs), in plants [1-4].

HMTs are mainly encoded by SET DOMAIN GROUP (SDG) and protein arginine methyltransferases (PRMTS) genes. Plant HMTs genes are involved in shoot and root branching, hormone regulation, morphogenesis, circadian cycle, fungal pathogens resistance, and ABA and salt stresses $[5,6]$. Histone methyltransferase-mediated process can be reversible by activating HDMs genes in plant. Two subfamily genes SWIRM and C-terminal domain (HDMA) and JmjC domain-containing proteins (JMJ) consist of $H D M s$ gene family [7]. Information about HDMs in plants revealed their functions in chromatin regulation, brassinosteroid (BR) signals, floral induction, pollen development, floral organ formation, and circadian 
cycle [8]. Four types of genes (HAGs, HAMs, HACs and HAFs) were identified in HATs gene family [9]. HATS genes are widely known in regulating transitions from vegetative to reproductive growth, abiotic and biotic responses, and stress-related hormone signals [10-12]. RPD3/HDA1 (HDA), Silent Information Regulator 2 (SRT) and HD2 (HDT) subfamilies make up of HDACs family [13]. Previous studies have proved that $H D A C s$ take part in vegetative and reproductive growth, stress adaptation, gene silencing, cell growth and regeneration $[14,15]$.

In the world, gramineous grain crops, including Triticum aestivum (T. aestivum), Hordeum vulgare (H. vulgare), Sorghum bicolor (S. bicolor), Setaria italica (S. italic) which is evolved from Setaria viridis (S. viridis) and Zea mays (Z. mays), are widely cultivated and provide abundant caloric intake for human [16]. In gramineae species, growth and development has proved to share closed relationships with grain yield and quality $[17,18]$. Biotic and abiotic stresses markedly affect crop development, yield and characters [19-23]. Although functions of $H M s$ in plant growth and environmental adaptions have been identified in some plant species. However, information regarding them in $T$. aestivum, $H$. vulgare, $S$. bicolor, S. viridis, S. italic and Z. mays is limited. The publications of these species genomes provides us useful tools to systematically characterize $H M s$ genes. Therefore, it is essential to investigate $H M s$ genes in these plants through bioinformatics approach.

In this study, 245, 72, 84, 93, 90 and $90 \mathrm{HMs}$ were respectively found in T. aestivum, H. vulgare, S. bicolor, $S$. viridis, $S$. italic and $Z$. mays genomes. Their location on chromosomes, conserved domains, evolution, synteny, promoter sequences and gene structures were analyzed. The expression patterns of TaHMs and $Z m H M s$ in developing wheat grain and maize seed were investigated, respectively. Moreover, the responses of TaHMs genes to growth regulators (BR, brassinazole (BRZ) and activated charcoal (AC)), and biotic as well as abiotic stresses (heat, drought, salt, insect feeding, nitrogen $(\mathrm{N})$ and cadmium (Cd)) were discussed. Moreover, expression profiles of $\mathrm{ZmHMs}$ were analyzed after gibberellin $\left(\mathrm{GA}_{3}\right)$ and drought treatment.

\section{Results}

\section{Identification and characterization of histone modification genes in $T$. aestivum, $H$. vulgare, S. bicolor, $S$. viridis, $S$. italic and $Z$. mays}

In Arabidopsis and rice, there were respectively 102 and 92 HMs, including 48 and $42 H M T s, 24$ and 24 HDMs, 12 and 8 HATs, and 18 and 18 HDACs (Fig. 1a). In total, 245, 72, 84, 93, 90 and 90 HMs were respectively identified in T. aestivum, H. vulgare, S. bicolor, S. viridis, S. italic and Z. mays (Fig. $1 \mathrm{a}$ and $1 \mathrm{~b}$ ). The number of HMTs, HDMs, HATs and HDACs were broadly equal among gramineae species, except for T. aestivum (Fig. 1a). Wheat HMs (HMTs, HDMs, HATs and HDACs) was severally about 2.4- and 2.7-folds as many as that of Arabidopsis and rice ones (Fig. 1a). HMTs, HDMs, HATs and HDACs could be separately classified into SDGs-PRMTs, HDMAs-JMJs, HAG-HAM-HAC-HAF, and HAD-SRT-HDT. There were 30 to 117 SDGs, 1 to 7 PRMTs, 3 to 12 HDMAs, 11 to $48 \mathrm{JMJs}, 1$ to 6 HAGs, 1 to 3 HAMs, 3-10 HACs, 1-6 HAFs, 11-32 HDAs, 1-6 SRTs and 1-5 HDTs among all species (Fig. 1b). Hexaploid wheat 
containing $A, B$ and $D$ sub-genomes, the $H M s$ on each wheat chromosome were counted to identify their distribution in each sub-genome. There were severally 3-8 T. aestivum SDGs (TaSDGs), 0-1 TaPRMTTaHAG-TaHAM-TaSRT-TaHDT, 0-2 TaHDMAs-TaHACs-TaHAFs, 1-4 TaJMJs, and 0-3 TaHDAs on chromosome 1A-7D (Fig. 1C). However, one TaHAG and TaSRT were located in unknown chromosome (Fig. 1c).

These gramineae $H M s$ were respectively named based on their chromosomal location (Fig. S1). Wheat chromosome 5A (Ta5A) contained the most of $H M$ s, followed by Ta2D (Fig. <link rid="fig1" $>1</$ link $>\mathrm{C}$ and S1-1). Most of barley HMs (HvHMs) were found in the longest chromosome 2 (chr2H), and HvSDG29, HvSDG30 and HvHDA14 were on unknown chromosome (Fig. S1-2). Sorghum SDGs (SbSDGs) were most numerous among all $H M s$ genes, 38 SbSDGs were distributed throughout nine chromosomes, and chromosome 2 contained most of SbHMs genes (Fig. S1-3). S. viridis HMs (SvHMs) were unevenly distributed on chromosomes 1-9; For example, there were 17 SvHMs genes on chromosome 1, while only two SVHMS (SVSDG36 and SVJMJ13) on chromosome 8 (Fig. S1-4). S. italic HMs genes were found from chromosome 1 to 9 , and chromosome 1 and 9 respectively shared the highest and smallest gene density (Fig. S1-5). Maize chromosome 1-10 respectively contained 4-13 maize HMs (ZmHMs) genes (Fig. S16). The detail information of gramineae HMs genes was list in Table S1. Their coding regions (CDS) lengths were from 195 (HvHDT3) to 7008 (AtSDG2) bp, with the deduced polypeptides ranged from 64 to 2335 amino acids (aa).

\section{Conserved domain and phylogenetic analysis of HMs genes}

Conserved domains of $H M s$ genes were investigated, various domains were presented in different kinds of HMs genes (Fig. S2). A total of 35 conserved motifs were identified in Arabidopsis and rice HMs proteins (Fig. S2-1). There were one to seven domains to be found in AtSDGs and OsSDGs proteins. For example, AtSDG7 only contained one SET_SETD2-like domain, three motifs (PHA03247, DUF4689 and SRA) were uncovered in OsSDG704, and OsSDG723 shared seven conserved domains, including SET_SETD1-like, ePHD_ATX1_2_like, PHD_ATX1_2_like, PWWP, FYRC, FYRN and TUDOR. Fifty-one elements were identified in TaSDGs, and most of these elements were the same as the ones discovered in AtSDGs and OsSDGs (Fig. S2-2). However, 15 specific elements, such as DUF5585, Jas, NupH_GANP, PostSET and so on, were identified in several TaSDGs. One to six conserved motifs were uncovered in each HvSDG protein, while we only found one distinctive motif (AMN1) in HvSDG26 compared with reference SDGs (AtSDGs and OsSDGs) (Fig. S2-3). Totally, 40 domains were identified in SbSDGs, and almost all of them were as the same as the ones identified in Arabidopsis and rice homologous proteins, while jas, CAF-1_p150, zf-TRM13_CCCH, GYF and PLN02983 were the exceptions (Fig. S2-4). Most of SvSDGs included more than two domains, and Jas, PRK12678, zf-TRM13_CCCH, Nucleo_P87, RSRP and GYF were characterized as peculiar motifs of SvSDGs (Fig. S2-5). All of SiSDGs contained two conserved domains, except for SiSDG1-2, SiSDG6-8, SiSDG10, SiSDG15, SiSDG20-21, SiSDG23, SiSDG26-29 and SiSDG33-34; And there were specific domains in SiSDG6 and SiSDG33 (Fig. S2-6). In maize SDGs, one to six conserved motifs were found, and eight distinctive (PRK07003, p450, jas, PHA03132, LbR-like, RSRP, F-box-like and HCP_like) domains were respectively identified in ZmSDG5, ZmSDG11, ZmSDG12, 
ZmSDG21, ZmSDG27 and ZmSDG39 (Fig. S2-7). About half of PRMTs proteins included PRMT_TIM, PRMT5 and PRMT_C domains, and all gramineaes PRMTs, except for ZmPRMT1, shared same structures with AtPRMT15 and OsPRMT708 (Fig. S2-8).

HDMAs contained one PLN03000, PLN02529, PLN02328, or PLN02976, respectively, and PLN02976 was the longest one (Fig. S2-9). Almost all of JMJs proteins included JmjC or JmjN, and 12-29 conserved structures were identified in both model and gramineae plants, totally (Fig. S2-10, 2-11, 2-12, 2-13, 214, 2-15 and 2-16). Specific motifs were found in gramineae JMJs. For example, there were respectively 14 distinctive domains in TaJMJ3, TaJMJ8, TaJMJ12, TaJMJ17, TaJMJ21, TaJMJ23, TaJMJ28, TaJMJ34, TaJMJ37, TaJMJ39, TaJMJ42, TaJMJ44 and TaJMJ48 (Fig. S2-11); zf-C5HC2 was characterized as barley peculiar motif in HvJMJ2 and HvJMJ3 (Fig. S2-12); Eight characteristic elements were identified in SbJMJ1, SbJMJ4, SbJMJ5, SbJMJ8, SbJMJ9 and SbJMJ17 (Fig. S2-13); CDS45 in SvJMJ1, ARID-TNG2-zf-RING_2 in SvJMJ8, cf-H2C2_2 in SvJMJ16 and ZZ in almost all of SvJMJs were identified as specific S. viridis motifs (Fig. S2-14); SiJMJ1 and SiJMJ9 contained several specific motifs (Fig. S2-15); And zf-H2C2 was identified as characteristic motif in ZmJMJ15 (Fig. S2-16).

There was Bromo_gcn5_like, COG5076, or ELP3 motif in all HAGs, except for AtHAG2, OsHAG704, SvHAG2, SvHAG4 and SiHAG1; And Hat1_N, NAT_SF, PLN02706 and acetyltransf_1 were also found in HAGs (Fig. S2-17). Only one domain (PLN00104) was found in all HAMs proteins (Fig. S2-18). Twelve conserved motifs, such as HAT_KAT11, PHD_HAC_like and ZnF_TAZ, were in HACs proteins (Fig. S2-19). All HAFs were made up of three to five conserved motifs, and they generally shared similar structures (Fig. S2-20).

Almost all of Arabidopsis and rice HDAs proteins contained one conserved domain (HDACs, Arginase_HDAC or Smc), while Smc was only found in AtHDA18 (Fig. S2-21). Like model plant HDAs proteins, all gramineae HDAs contained one HDACs or Arginase_HDAC domain, while LRAT was also in ZmHDA10 and ZmHDA11 (Fig. S2-22, 2-23, 2-24, 2-25, 2-26 and 2-27). One SIRT4, SIRT7 or SIR2 domain was respectively uncovered in each SRT protein (Fig. S2-28). All HDTs proteins, except for HvHDT1, HvHDT3 and ZmHDT1, included NPL domain; While other domains (PRK13108, ZnF_U1, lambda-1, zf-C2H2_jaz, TF『F_alpha, PRK13808 and zf-C2H2_6) were also found in several HDTs proteins (Fig. S2-29).

In order to identify evolutionary relationships of $H M s$ genes, unrooted phylogenetic trees were constructed (Fig. S3). AtHMTs (except AtSDG41), OsHMTs and TaHMTs were classed into groups A-E which can be further subdivided (Fig. S3-1). For example, AtPRMTs, OsPRMTs and TaPRMTs were in class B; TaPRMTs, AtPRMT15 and OsPRMT708 were clustered in subclass b1, and other ones were found in subclass b2. All Arabidopsis, rice and barley HMTs genes were classified into eight groups. All SDGs were clustered together in class A-D and F-H, and PRMTs were divided into subgroup e1 and e2 in group E (Fig. S3-2). In Fig. S3-3, AtPRMTs, OsPRMTs and SbPRMTs were clustered together in group A which could be divided into subgroup a1 and a2, and SDGs (except for AtSDG41) were identified in groups B-G. AtPRMTs, OsPRMTs and SvRMTs were grouped in either class A or B, and the other HMTs were in class C-H (Fig. S3- 
4). All Arabidopsis, rice and S. italic SDGs got together in group B-E, with exceptions of OsSDG738, SiSDG17 and SiSDG34, and PRMTs were all in group A (Fig. S3-5). AtPRMTs_OsPRMTs_ZmPRMTs and AtSDGs_OsSDGs_ZmSDGs were severally clustered together in group A and B-G (Fig. S3-6). In class A, B and $D$, model and gramineae JMJs closely got together, and HDMAs were divided into subclass $\mathrm{c} 1$ and $\mathrm{c} 2$ in group C (Fig. S3-7). The evolutionary relationship of HATs was investigated in Figure S3-8, HAGs were in group $B, C$ and $E$, and HAFs, HAMs and HACs were separately divided into group $A, D$ and $F$. HDTs and SRTs were severally clustered into group $A$ and $B$, while HDAs were found in group $C$ and D (Fig. S3-9).

\section{Synteny analysis among $H M s$ genes}

In order to identify expansion patterns of $H M s$ genes, duplicated blocks within each gramineae genome were investigated (Fig. S4). Totally, 144 pairs of TaHMs were identified from 21 chromosomes (Fig. 2a and Fig. S4-1). There were respectively 72 TaSDGs, 2 TaPRMTs, 8 TaHDMAs, 30 TaJMJ, 1 TaHAG, 2 TaHAM, 3 TaHAC, 3 TaHAF, 20 TaHDA and 3 TaSRT gene pairs (Fig. 2b). Only 4 SbHMs gene pairs, including SbSDG16-SbSDG37, SbSDG22-SbSDG26, SbJMJ1-SbJMJ10 and SbHDA11-SbHDA5, were identified in S. bicolor genome (Fig. 2a, Fig. 2b and Fig. S4-2). A total of four types of SvHMs genes (4 SVSDGs, 2 SVJMJS, 1 SVHACs and 2 SVHDAs) pairs were found (Fig. 2a, Fig. 2b and Fig. S4-3). Four pairs of SiSDGs, two pairs of SiJMJs and one pair of SiHACs were characterized (Fig. 2a, Fig. 2b and Fig. S4-4). In 10 chromosomes, we found fourteen $\mathrm{ZmHMs}$ gene (6 ZmSDGs, 2 ZmJMJs, 1 ZmHAGs, 1 ZmHAMs, 2 $Z m H A C s$ and $2 Z m H D A s$ ) pairs (Fig. 2a, Fig. 2b and Fig. S4-5). However, no HvHMs gene pairs were identified (Fig. 2a and Fig. 2b).

We investigated syntenic relationship of gramineae and Arabidopsis HMs (Fig. 2c and Fig. S5). One HMTs (AtSDG24 and TaSDG97), four HDMs (AtJMJ13 and TaJMJ3, AtJMJ13 and TaJMJ7, AtJMJ13 and TaJMJ11, AtJMJ13 and TaJMJ42) and one HDACs (AtHDA9 and TaHDA12) gene pairs were respectively identified between Arabidopsis and wheat (Fig. 2c and Fig. S5-1). Only AtSDG24 and SbSDG19 was found in the same gene pair (Fig. 2c and Fig. S5-2). Three HMs gene pairs were respectively characterized in Arabidopsis-S. viridis (AtSDG24 and SvSDG2, AtJMJ13 and SvJMJ2, AtHDA5 and SvHDA4) and Arabidopsis-S. italic (AtSDG31 and SiSDG4, AtJMJ13 and SiJMJ12, AtHDA10 and SiHDA10) (Fig. 2c, Fig. S5-3 and Fig. S5-4). While no HMs gene pairs were identified in Arabidopsis-barley and Arabidopsismaize.

All kinds of HMs gene pairs were found between rice and wheat genome, including 62 pairs of HMTs (59 pairs of $S D G s$ and 3 pairs of PRMTs), 25 pairs of HDMs (9 pairs of HDMAs and 16 pairs of JMJs), 8 pairs of HATs (1 pairs of HAGs, 3 pairs of HAMs, 3 pairs of HACs and 3 pairs of HAFs) and 16 pairs of HDACs (12 pairs of HDAs, 2 pairs of SRTs and 2 pairs of HDTs) (Fig. 2d, Fig. 2e and Fig. S6-1). A total of 27 pairs of OsHMs-HvHMs were identified, such as OsSDG713-HvSDG3, OsPRMT708-HvPRMT1, OsHDMA701HvHDMA1, OsJMJ718-HvJMJ8, OsHAM701-HvHAM1, OsHAC703-HvHAC3, OsHAF701-HvHAF1, OsHDA712-HvHDA2, OsSRT702-HvSRT2 and OSHDT701-HvHDT1 (Fig. 2d, Fig. 2e and Fig. S6-2). Nine of eleven kinds of $H M s$ gene pairs were found between $S$. bicolor and rice, including twenty-one pairs of $S D G s$, three pairs of $H D M A s$, ten pairs of $J M J s$, two pairs of $H A G s$, one pair of HACs, PRMTs as well as 
HAFs, 6 pairs of HDAs and 2 pairs of HDTs (Fig. 2d, Fig. 2e and Fig. S6-3). There were 41 pairs of HMs between $S$. viridis and rice, including most kinds of $H M s$ (Fig. 2d, Fig. 2e and Fig. S6-4). Orthologous pairs were identified in all HMs between S. italic and rice, except for HAGs (Fig. 2d, Fig. 2e and Fig. S6-5). More than 50 pairs of maize and rice HMs were found through comparison of their genomes (Fig. 2d, Fig. 2e and Fig. S6-6).

In order to evaluate selection pressure during duplication of above gene pairs, their $\mathrm{Ka}$, $\mathrm{Ks}$ and $\mathrm{Ka} / \mathrm{Ks}$ values were calculated. The data showed that $\mathrm{Ka} / \mathrm{Ks}$ values were all less than or generally equal than 1 (Table S2-4). However, several gene pairs, such as SiJMJ5-SiJMJ19, AtJMJ13-TaJMJ3, and AtJMJ13TaJMJ7, shared no nonsynonymous mutation according to their Ks values.

\section{Promoter and structure analyses of $H M s$ genes}

$H M s$ genes played important roles in plant stress and defense responses [24, 25]. Therefore, we identified stress-related elements in gramineae HMs genes (Fig. S7). In TaHMTs, TaHDMs and TaHDACs genes, at least one abscisic acid-, MeJA-, defense-, drought-, low temperature- or salt-related elements were uncovered (Fig. S7-1, 2 and 4). Abscisic acid, MeJA, and defense responsiveness elements were also identified in TaHATS genes (Fig. S7-3). There were two to thirteen stress-related motifs (defense and stress, abscisic acid and MeJA-responsiveness elements) in HvHMTs, But no defense and stress responsiveness cis-elements were uncovered in all HvHDMs, except for HvJMJ10 and HvJMJ11 (Fig. S75). SbSDG3, SbSDG13, SbPRMT1 and SbJMJ16 only contained one defense and stress, abscisic acid or MeJA-responsiveness motif, and other SbHMs included more than two stress-related elements (Fig. S7-6). Like $S b H M s$, numbers of stress, abscisic acid or MeJA-related elements were identified in $S$. viridis and $S$. italic HMs (Fig. S7-7 and S7-8). In ZmHMs, stress-related (MeJA-responsive, drough-inducibility, abscisic acid responsive, salicylic acid responsive, low-temperature responsive, defense and stress responsive, and wound-responsive), gibberellin-responsive and seed-specific regulation elements were identified (Fig. S7-9).

We identified $H M s$ gene structures. In general, homologous $H M s$ genes, especially genes in same pairs, shared similar structures, but genes lengths in different groups were various (Fig. S8). Regarding TaHMTs, most of homologous genes were made up of more than one CDS, and most of TaHMTs gene lengths were more than 3000 bp (Fig. S8-1). TaJMJs genes can be broadly divided into two classes, all TaHDMAs were in the same group; there were one to nine CDSs in TaHDMAs, and TaJMJs generally shared more CDSs than TaHDMAs, And all TaHDMs were about 15000 bp length, with the exceptions of TaJMJ31 and TaJMJ34 (Fig. S8-2). In terms of TaHATs, genes in each cluster contained relatively consistent structures, and TaHAG2 were longer about 7000 bp than other genes for its long non-coding sequence (Fig. S8-3). All TaHATs, except for TaHDA18 and TaHDA23, were segmented by non-coding region, and they were shorter than other TaHMs (Fig. S8-4). All HvHMTs, except for HvSDG4, shared short non-coding sequence, and most of them were about 2000 to 5000 bp (Fig. S8-5). Four HvHDMs (HvJMJ4, HVHDMA1, HvHDMA2 and HvHDMA4) only contained one CDS, while other ones were separated, and most of them (except for HvJMJ6) were about 9000 bp length (Fig. S8-6). Short CDSs were identified in 
HvHAT and HvHDAC genes, most of HvHAT and HvHDAC genes were about 1000 bp, while HvHAG1, HvHAF1, HvHDA3, HvHDA8 and HvHDT1 were obviously long than other genes (Fig. S8-7 and Fig. S8-8). Lots of SbHMTs (SbSDGs and SbPRMTs), SbHDMs (SbHDMAs and SbJMJs), SbHATs (SbHAGs, SbHAMs, SbHACs and SbHAFs) and SbHDACs (SbHDAs, SbSRTs and SbHDTs) were made up of short CDSs, while several genes contained one to two long CDSs (Fig. S8-9, 8-10, 8-11 and 8-12). About a third of SbSDGs included one long CDS; And all SbHMTs were less than 20000 bp, except for SbSDG7 (Fig. S8-9). SbHDMs were about from 2900 to 23000 bp long, and their CDSs and UTRs were generally short (Fig. S810). SbHATs and SbHDACs were respectively shorter than SbHMTs and SbHDMs, they were separated by short non-coding sequences, while several SbHDACs (SbHDA7-8 and SbSRT1) contained long non-coding sequences (Fig. S8-11 and 8-12). Numbers of short CDSs were found in many SVHMTs and SVHDMS; And several genes, especially SvSDG7-8, SVSDG16, SvSDG18, SVSDG30, SVHDMA3-4, SVJMJ2, SvHAC4 and SVHDA3, shared long CDS and UTR (Fig. S8-13 and 8-14). SVHATs and SVHDACs were generally short in gene and CDS length, while UTRs in several genes were long (Fig. $<$ link rid="fig8">8</link $>-15$ and 8-16). Most of SiHMs contained numbers of CDSs, while only one to two long CDSs were in SiSDG18, SiSDG31, SiSDG41, SiSDG5, SiSDG11, SiSDG35, SiSDG27, SiHDMA1 and SiHDMA3 (Fig. S8-17, 8-18, 8-19 and 8-20). ZmHMs (except for ZmSDG1, ZmSDG3, ZmSDG5, ZmSDG16, ZmSDG18, $Z m S D G 28-29, Z m S D G 36, Z m S D G 41, Z m H D M A 2$ and $Z m H D A 3)$ were made up of either lots of short CDSs or one to four long ones, and one long 5' or 3' UTR were in ZmSDG31, ZmJMJ9, ZmHDMA1, ZmHAC1, ZmHAC3-4, ZmHDA3 and ZmHDA5 (Fig. S8-21, 8-22, 8-23 and 8-24).

\section{The expression patterns of TaHMs in developing wheat grain, response to brassinosteroid and activated charcoal}

In order to investigate potential roles of $H M s$ in wheat grain growth and development, their expression profiles were generated from endosperm, inner pericarp and outer pericarp (Fig. 3). In cluster 1, TaSDG53, TaSDG29, TaSDG56 and TaSDG61 shared high expression levels in all tissues, especially in inner pericarp; Several TaSDGs in cluster 2, such as TaSDG15, TaSDG103 and TaSDG21, also highly expressed in inner pericarp; In cluster 3, seven TaSDGs showed relatively low level in outer pericarp; TaSDGs in cluster 4 and 5 shared lower expressions than genes in other clusters; In cluster 6, most of genes were highly expressed in inner or outer pericarp (Fig. 3a). The expression level of TaHDMAs and TaJMJs were generally low than other type genes (Fig. 3b). Genes in cluster 1 mainly expressed in endosperm or outer pericarp, while expression of several genes, particularly TaJMJ5, TaJMJ9, TaJMJ21 and TaJMJ39, were very low; Levels of TaHDMs were higher in cluster 2 and 3 than 1, transcription levels of about half of TaHDMs (especially TaHDMA3, TaJMJ47, TaJMJ27, TaJMJ35 and TaJMJ41) were high in inner or outer pericarp, and TaJMJ12 and TaJMJ47 were mainly detected in endosperm (Fig. 3b). TaHATs can be classified into two classes according to their expression patterns; And genes in cluster 1 were more highly expressed in all tissues than that in cluster 2 (Fig. 3c). In terms of TaHDACs, TaHDA22, TaHDA31, TaHDA28, TaHDA10 and TaHDA20 in cluster 1 shared lower expression levels in all grain tissue layers than other TaHDACs genes in cluster 2-4; in cluster 2, TaHDA4 and TaHDA6 were mainly present in endosperm, TaHDA16-17 showed high level in outer pericarp, and TaHDA29, TaHDA19 and TaSRT1 were mainly detected in inner pericarp; In cluster 3 and 4, TaHDAs, TaSRT3 and TaSRT5 were highly expressed in pericarps (Fig. 3d). 
In Arabidopsis, rice, wheat and maize, BR has been proved to play important roles in root growths, including lateral root (LR) initiation and hair formation [26-29]. BR treatment significantly increases wheat lateral root number, but inhibits root length and root diameter, and BR synthesis inhibitor BRZ showed opposite roles on lateral root number and root diameter [26]. HMs genes have been known to regulate various developmental processes, while their information in regulating wheat root is absent. In this study, we analyzed their expression profiles during BR- and BRZ-mediated root growth (Fig. 4). In cluster 2, TaSDG4, TaSDG23, TaSDG55 and TaSDG112 were obviously induced more than 2-fold by BRZ, but BRZ repressed TaSDG26, TaSDG68, TaSDG89, TaSDG92, TaSDG95, TaSDG103 and TaJMJ5 in cluster 1 (Fig. 4a). BR treatments increased more than ten TaHMs (especially TaJMJ5 and TaSDG28) expressions, but several TaHMs were repressed by BR1 or BR2 (Fig. 4b and c). For example, TaSDG26, TaSDG28 and TaJMJ5 were induced by both BR1 and BR2 treatments; TaSDG92 and TaSDG101 were specifically up-regulated by BR2; And transcription levels of TaJMJ21, TaSDG53 and TaHDA18 were repressed in both BR1 and BR2 groups.

In plant culture, $\mathrm{AC}$ is widely used to promote seedling growth, and its effect on growth of wheat seedlings has been identified [30]. AC treatment distinctly promotes wheat seedlings growth rate, accompanied by increasing soluble protein, root activity, total phenol and sugar content [30]. We found that 26 and 31 TaHMs genes were differentially expressed in root and leaf after AC treatment, respectively (Fig. 5). In root and leaf, about half of these TaHMs were down-regulated or up-regulated by AC. For example, TaSDG68 and TaSDG84 were respectively decreased about 4- and 8-folds in root and leaf after AC treatment, TaSDG55 in root was evidently increased in AC treatment group, and TaJMJ21 was up-regulated by $A C$ in leaf (Fig. $5 a$ and $5 b$ ).

\section{The responses of TaHMs to abiotic and biotic stresses}

In order to explore whether TaHMs respond to abiotic stresses, their expression analyses were analyzed after HS, DS and HD treatments using published RNA-seq [31]. We found 86 TaHMTs (83 TaSDGs and 3 TaPRMTs) genes were differentially expressed at 1 or $6 \mathrm{~h}$ after heat, drought or heat plus drought treatments (Fig. 6a). These TaHMTs genes were able to be divided into 6 clusters; In cluster 1, almost all TaSDGs were respectively induced and repressed by DS at 1 and $6 \mathrm{~h}$, and up-regulated by HD at $6 \mathrm{~h}$; About 20 TaSDGs in cluster 2 were obviously increased at $6 \mathrm{~h}$ after HS and HD treatments, while decreased at $1 \mathrm{~h}$, and several TaSDGs were clearly induced or inhibited by DS; In cluster 3, TaSDGs were generally induced by both HS and HD at $6 \mathrm{~h}$, while they were suppressed at $1 \mathrm{~h}$ in HS and HD groups, and DS increased these genes at 1 or $6 \mathrm{~h}$; Eleven TaSDGs in cluster 4 were mainly increased by DS, but decreased by HD at $1 \mathrm{~h}$; In cluster 5, TaSDG 117 was respectively repressed at $6 \mathrm{~h}$ after HS treatment and induced by HD at $1 \mathrm{~h}$, and other genes were obviously up-regulated at $6 \mathrm{~h}$ by DS; In cluster 6, TaSDG28 and TaSDG53 were mainly induced by HS at $1 \mathrm{~h}$, while they were inhibited at $1 \mathrm{~h}$ after HD treatment, and DS upregulated other genes at $1 \mathrm{~h}$. All TaHDMs were divided into four clusters (Fig. 6b). Expression of TaJMJ21 was high after HS and HD treatment, and it was increased by DS at $1 \mathrm{~h}$; In cluster 2, TaJMJ7, TaJMJ11 and TaJMJ3 were generally up-regulated by DS and $\mathrm{HD}$ at $1 \mathrm{~h}$, and other genes were generally up-regulated at $6 \mathrm{~h}$ after HS and HD treatments; TaHMTs in cluster 3 were clearly induced by DS, and 
several genes were induced by HS or HD at $6 \mathrm{~h}$; In cluster 4, TaJMJ40 was respectively increased by HS at $6 \mathrm{~h}$ and $\mathrm{HD}$ at $1 \mathrm{~h}$, and TaJMJ9 was increased at $6 \mathrm{~h}$ after HS treatment. TaHATs were clustered into 2 classes (Fig. 6c). TaHAG1, TaHAG2 and TaHAG5 were increased after HS and HD treatment in cluster 1; In cluster 2, TaHAM2 and TaHAM3 were obviously up-regulated at $6 \mathrm{~h}$ in HS and HD groups, and other genes were induced by HS, DS or HD at least one time point. As show in Fig. 6d, TaHDA4, TaHDA17 and TaSRT2 were induced by DS in cluster 1, and TaHDA4 was also increased in HS group; DS treatment increased 10 TaHDACs expression in cluster 2, and these genes were also affected by HS or HD at several time points; Nine TaHDACs were distinctly induced by HS, DS or HD in cluster 3; And TaHDACs (TaHDA10, TaHDA12, TaHDA16, TaHDA19 and TaHDA21) in cluster 4 were primarily expressed at $6 \mathrm{~h}$ in HS and HD groups.

To investigate responses of TaHMs to SS, their expression profiles were identified in salt sensitive wheat cultivar CS and insensitive cultivar QM after SS treatment. After SS treatment, almost all TaHMs were upregulated at least one time point (Fig. 7). TaHMTs can be clustered into three clusters (Fig. 7a). In cluster 1, TaSDG17 and TaSDG21 were significantly induced at $12 \mathrm{~h}$ after SS treatment in QM. TaSDGs and TaPRMTs in cluster 2 were up-regulated at several points in either CS or QM group after SS treatment. For example, TaSDG76 was obviously induced at 6 and $12 \mathrm{~h}$ after SS treatment, and at 12 and $24 \mathrm{~h}, T a S D G 2$ and TaSDG11 were respectively increased by SS treatment in CS and QM. At most of time points, TaSDGs genes were visibly induced by SS in cluster 3. For example, expression level of TaSDG92 was increased at 6 and $12 \mathrm{~h}$ after SS treatment in CS, and it was induced by SS from 12 to $48 \mathrm{~h}$ in QM; SS up-regulated TaSDG28 at all time points in both of cultivars (Fig. 7a). In terms of TaHDMs, most of genes were upregulated by SS from 6 to $24 \mathrm{~h}$ in cluster 1 ; Genes in cluster 2 were induced by SS treatment at most of time points; Transcript of TaJMJ18, TaJMJ27, TaJMJ23, TaJMJ25, TaJMJ38, TaJMJ44 and TaJMJ48 were increased from 12 to $48 \mathrm{~h}$ after SS treatment in cluster 3; In both CS and QM, there were six TaJMJS to be obviously induced by SS in cluster 4 (Fig. 7b). TaHATs were divided into two clusters (Fig. 7c). For example, in cluster 1, TaHAC8 and TaHAC10 were mainly regulated by SS in CS, and TaHAF4, TaHAG3 and TaHAC6 were induced by SS in both CS and QM; In cluster 2, SS treatment generally increased expression levels of TaHACs and TaHAGs, especially TaHAC1 and TaHAC2, at all time points (Fig. 7c). Expressions of TaHDACs, especially genes in cluster 3, were also clearly increased at least one time point after SS treatment (Fig. 7d).

Sitobion avenae (S. avenae) and Schizaphis graminum (S. graminum) are two main wheat insect pests which will lead to obvious yield losses [32]. Compared with non-phytotoxic aphid $S$. avenae, phytotoxic aphid $S$. graminum feeding causes more severe damage in wheat leaves [33]. Nitrogen $(N)$ is an essential macronutrient for plant growth and development, and low $\mathrm{N}$ stress obviously repressed wheat leaf and root growth [33]. Cadmium (Cd) highly inhibits leaf photosynthesis, carbon and nitrogen metabolism, and wheat growth and yield [34]. In order to provides information for TaHMs in respond to biotic, nutrition and heavy metal stresses, expression patterns of TaHMs were identified in above transcriptome researches [32-34]. In wheat leaves, TaJMJ7 was increased about 3.4- and 4-fold after S. avenae and S. graminum feeding, respectively; Another TaJMJ gene which was induced by S. avenae infection is TaJMJ11; Transcription levels of TaJMJ40 and TaJMJ42 were higher in S. graminum feeding group than control 
one; While TaHDA17, TaSDG73, TaHDA20, TaSDG81, TaHDA22 and TaSDG89 were distinctly controlled by S. graminum feeding (Table 1). N stress obviously suppressed TaSDG73 and TaHDA20 expression in leaf, while it up-regulated TaJMJ11 and TaJMJ3 in root (Table 1). In roots, Cd induced 12 TaHMs, such as TaSDG13, TaJMJ28 and TaHDT1, about 2.2- to 6.4-folds, but decreased TaSDG102 expression (Table 1).

\section{The diverse responses of TaHMs to growth and stress signals}

In order to investigate multiple functions of TaHMs in wheat growth and stresses adaption, a Venn diagram was constructed with above identified DEGs (Fig. 8). DEGs can be clustered into six sets, including BR or BRZ (BR_BRZ) class, AC class, heat or drought (Heat_Drought) class, salt class, S. avenae or S. graminum (Sa_Sg) class, and $\mathrm{N}$ or Cd (N_Cd) class (Fig. 8 and Table S5). Two TaHMs (TaSDG68 and TaJMJ5) were commonly in response to BR_BRZ and AC; Expression patterns of TaSDG95 and TaSDG103 were altered by both BR_BRZ and salt treatments; Two DEGs (TaHDA4 and TaHAG5) were identified after $A C$ and Heat_Drought treatments; The expressions of three TaSDGs (TaSDG27, TaSDG35 and TaSDG84) and TaJMJ25 were commonly induced or repressed by AC or salt; TaSDG102 was differently expressed in both AC and N_Cd groups; A total of $72 \mathrm{TaHMs}$ simultaneously respond to heat, drought or salt treatments; TaSDG81 and TaJMJ7 were simultaneously uncovered as DEGs in Heat_Drought and Sa_Sg classes, when comparing with control group; TaSDG101 and TaJMJ21 were differently expressed after BR_BRZ, AC and Heat_Drought treatments; After BR_BRZ, AC and salt treatments, two genes (TaSDG55 and TaJMJ17) were identified as DEGs; There were 21 DEGs, such as TaSDG1, TaJMJ13, TaHAC2, TaHDA6 and so on, to be found in AC, Heat_Drought and salt classes at the same time; TaSDG2 and TaSDG4 expressions were affected by BR or BRZ, AC, heat or drought and salt stress; TaJMJ40 and TaJMJ42 were detected to respond to AC, heat or drought, salt, and S. avenae or $S$. graminum, commonly; We found that TaSDG13 and TaJMJ28 were common DEGs after AC, Heat_Drought, Salt and N_Cd treatments; Only one gene TaJMJ11 was in response to Heat_Drought, salt, Sa_Sg, and N_Cd, simultaneously; TaJMJ34 was commonly induced or repressed by five signals, including BR_BRZ, AC, Heat_Drought, Salt.and N_Cd (Fig. 8 and Table S5).

\section{Expression analysis of $\mathrm{ZmHMs}$ in developing seed and response to GA treatment}

To investigate functions of $Z m H M s$ in maize growth and development, expression profiles of $Z m H M s$ were analyzed in different seed growth stages of B73 and SWL01 (Fig. 9a and b). SWL01 is a mutant of $\mathrm{B} 73$ and contain higher viscosity than B73 [35]. From 0 to $24 \mathrm{~d}$ after pollination (DAP), $80 \mathrm{ZmHM}$ genes were clustered into 5 classes (Fig. 9a). During whole experiment period (especially at 2 DAP), ZmSDG36 in cluster 1 shared higher expression level than genes in other classes; In cluster 2, ZmHMs shared higher expression at early stages (from 0 to 8 DAP) than late periods (from 16 to 24 DAP); Elven ZmHMs highly expressed at all stages, especially from 0 to 4 DAP, in cluster 3; Transcription level of ZmHMs in cluster 4 generally decreased over time, with the exceptions of ZmJMJ12, ZmSDG11, ZmSDG3 and ZmHDA13; Compared with genes in other clusters, ten $Z m H M$ genes (especially $Z m S D G 16$ ) were weekly expressed from 0 to 24 DAP in cluster 5 . There were a total of $81 \mathrm{ZmHM}$ genes to be detected during SWL01 seed development (Fig. 9b). Like genes in B73, these ZmHMs were distributed into 5 clusters in SWL01; 
$Z m H M s$ in cluster 1 and 2 (especially in cluster 2) mainly expressed at 0, 2 and 4 DAP; ZmHMs, such as $Z m S D G 29, Z m S D G 36, Z m S D G 40$ and $Z m H D A 1$, shared higher expression level in cluster 3 than genes in other clusters, while transcription levels of genes in cluster 4, especially ZmSDG5, ZmSDG14, ZmSDG16, $Z m S D G 19, Z m J M J 4$ and $Z m H D T 1$, were generally low; In cluster 5, most of $Z m H M s$ gradually decreased over time, but ZmJMJ12, ZmSDG11, ZmSDG41 and ZmSDG6 showed opposite trend. A total of 79 $Z m H M s$ were commonly expressed in both B73 and SWL01 seeds, but most of them shared different expression patterns in two cultivars. For example, expression level of ZmSDG41 was higher in B73 than SWL01; ZmHAF1 were gradually decreased over time in B73, while its expression was almost no change in SWL01 (Fig. 9c). ZmSDG23 was specifically expressed in B73, and ZmJMJ4-ZmSDG14 were only detected in SWL01 (Fig. 9c). GA 3 application significantly promoted leaf sheaths growth of D11 [36]. Seven $Z m H M$ genes were differently expressed between GA and control groups (Fig. 9d). In cluster 1 , $Z m H D M A 3, Z m H D A 10, Z m J M J 10$ and $Z m S D G 10$ were down-regulated by GA, but $Z m H D A 12, Z m H D A 3$ and $Z m S D G 33$ were up-regulated.

\section{Expression analysis of $\mathrm{ZmHMs}$ in response to drought stress}

To identify potential roles of $Z m H M s$ in drought adaption, their expression patterns were analyzed in drought tolerant cultivars (ND476 and H082183), drought sensitive cultivars (ZX978 and Lv28), and C7-2 (Table 2). Totally, ten $Z m H M s$ were identified as DEGs in response to drought stress. After drought treatment, transcription level of $Z m J M J 2$ was increased about 6-fold in ND476 than ZX978, but $Z m H D A 11$ was repressed in ND476. ZmSDG5, ZmJMJ4 and ZmSDG24 were induced by drought treatment in C7-2, but ZmSDG33 and ZmJMJ17 were controlled. In Lv28 and H082183, ZmJMJ5 was upregulated by both moderate and severe drought treatments. Expression level of ZmSDG1 was increased in H082183 after moderate drought treatment, while $Z m H D A 2$ was obviously down-regulated after severe drought treatment.

\section{Discussion}

The essential functions of $H M s$ on plant growth, biotic and abiotic stresses have been reported in model plants, and previous studies have made great advances on HMs. However, little information has been reported in gramineaes. Here, we systematacially characterized TaHMs, HvHMs, SbHMs, SvHMs, SiHMs and $Z m H M s$ genes. Their important information, including gene location, conserved domain, gene phylogeny, gene expansion as well as synteny, cis-elements in promoter, and gene structures, were characterized. Moreover, their expression analysis during wheat and maize growth and stress adaptions were investigated. These findings will provide basis for further functional analyses of $H M s$ genes.

\section{Comparison of HMs genes between gramineae and model plants}

In model plants, identifications of HMs, including HMTs (SDGs and PRMTs), HDMs (HDMAs and JMJs), HATs (HAGs, HAMs, HACs, and HAFs), and HDACs (HDAs, SRTs, and HDTs), have been completed. In Arabidopsis, 48 AtHMTs, 24 AtHDMs, 12 AtHATs and 18 AtHDACs were found [10, 13]. There were 92 
OsHMs, including 42 OsHMTs, 24 OsHDMs, 8 OsHATs and 18 OsHDACs [37]. In six gramineae plants, 245 TaHMs (120 TaHMTs, 60 TaHDMs, 24 TaHATs and 41 TaHDACs), 72 HvHMs (31 HvHMTs, 15 HvHDMs, 7 HvHATs and 19 HvHDACs), 84 SbHMs (39 SbHMTs, 21 SbHDMs, 7 SbHATs, 17 SbHDACs), 93 SvHMs (41 SvHMTs, 22 SvHDMs, 12 HvHATs and 18 SvHDACs), 90 SiHMs (43 SiHMTs, 24 SiHDMs, 7 SiHATs and 16 SiHDACs) and $90 \mathrm{ZmHMs}$ (42 ZmHMTs, $20 \mathrm{ZmHDMs}, 10 \mathrm{ZmHATs}$ and $18 \mathrm{ZmHDACs}$ ) were identified, respectively (Fig. 1). In terms of gene number, TaHMs were respectively about 2.4- and 2.6-folds greater than AtHMs and OsHMs, And TaSDGs, TaHDMAs, TaJMJs, TaHAGs, TaHAMs, TaHACs, TaHAFs, TaHDAs and TaSRTs were expanded 1.5 to 3 folds; However, the number of other species HMs genes slightly varied when comparing with the model ones (Fig. 1a and 1b). In wheat, a total of 144 gene pairs were identified in 10 of 11 kinds $H M s$ genes, but there were 4 to $14 \mathrm{HMs}$ gene pairs among S. bicolor, S. viridis, $S$. italic and $Z$. mays, and no gene duplication was identified in $H$. vulgare. Genome duplication appeared during evolution of species [38], and wheat genome which contains three homologous subgenomes [16]. Therefore, these expansions of wheat $H M s$ genes may be associated with gene and genome duplications during evolution.

In general, gramineae, Arabidopsis and rice HMs genes shared similar domains (Fig. S2). However, there were several exceptions. For example, TaSDGs, HvSDGs, ZmSDGs, TaJMJs and SbJMJs respectively contained 15, 2, 10, 14 and 8 special motifs (Fig. S2-2, 2-3, 2-7, 2-11 and 2-13). New functions can be predicted from unique domains. Therefore, we should pay more attention to these genes sharing special elements in future. According to phylogenetic analysis, each type of $H M s$ was clustered together (Fig. S3). However, there were exceptions. For example, AtSDG41, HvSDG4, SiSDG17, SiSDG34 and OsSDG738 shared closed relationship with PRMTs other than SDGs (Fig. S3-1, 3-2, and 3-5). This may due to their incompletely matching protein sequences.

To better understand information about gramineaes $H M s$, duplicated blocks between model plants and gramineaes were determined. In this study, 13 orthologous genes were identified between Arabidopsis and six gramineae species (Fig. S5 and Table S3)), and 389 rice-gramineae gene pairs were found (Fig. S6 and Table S4), indicating these genes in pairs shared common ancestors. The gene pair showed huge difference between Arabidopsis-gramineae and rice-gramineae in terms of number, which may be due to diversity in evolutionary history of monocotyledons and dicotyledons. A number of AtHMs and OsHMs were reported to be involved in plant growth and stress responses $[5,6,8,10-12,14,15,39-41]$. According to model plant orthologous genes, lots of unknown gramineae $H M s$ can be inferred. However, these predictions must be confirmed in further experiments. Gene evolution mode can be determined through $\mathrm{Ka} / \mathrm{Ks}$ values. The $\mathrm{Ka} / \mathrm{Ks}$ ratios of all gene pairs identified in this study were all less than 1 , indicating their purifying selection [42].

\section{The potential functions of TaHMs and $\mathrm{ZmHMs}$ genes in plant growth and stress responses}

Like transcription factors, $H M s$ are important regulators referring to a lot of biological processes, including plant growth and development. We proposed that TaHMs and $Z m H M s$ shared similar roles with known HMs. Candidate TaHMs genes involved in wheat developing grain, and $Z m H M s$ genes referring to 
maize seed development were characterized in our study. The expression patterns of TaHMs genes showed that almost all of TaHMs (especially TaSDGs in cluster 1 (Fig. 3a), TaHDMs in cluster 3 (Fig. 3b), TaHATs in cluster 1 (Fig. 3c) and TaHDACs in cluster 4 (Fig. 3d)) were expressed in developing wheat grains, and many genes were highly expressed in specific grain tissues layer (Fig. 3). About eight over ten $Z m H M s$ were expressed with different patterns in developing maize seeds (Fig. 9a and b). ZmHM genes which specifically expressed in B73 (ZmSDG23) or SWL01 (ZmSDG14 and ZmJMJ4) were found (Fig. 9c). In addition, some common expressed ZmHMs between B73 and SWL01showed various expression patterns (Fig. 9c). Moreover, seed-specific motifs of $Z m H M s$ were identified. These findings suggest that TaHMs genes affect grain growth and development, most of $Z m H M$ genes play roles in waxy and regular maize seeds development, and several $Z m H M s$ specifically take part in regulating seeds viscosity.

BR is an essential plant hormone and stimulates wheat root hair formation and LR initiation [26]. However, responses of TaHMs to BR and BRZ were not identified. The findings in this study showed that four TaSDGs were induced by BRZ, but six TaSDGs, TaJMJ5 and TaHDA14 were repressed (Fig. 4a); And BR respectively increased or decreased 11 TaHMs genes expression (Fig. 4b). GA treatment stimulates leaf sheath elongation of maize seedlings and altered seven ZmHMs expression (Fig. 9d). The above results indicated these TaSDGs and $Z m S D G s$ were likely to be involved in BR-mediated root growth and GA-mediated leaf development. AC has been proved to be a positive growth regulator in wheat culture [30]. However, relationship between TaHMs and AC was not reported until now. Totally, $26 \mathrm{TaHMs}$ genes were differentially expressed between control and $A C$ treated roots, and about half of them were respectively repressed or induced by AC (Fig. 5a). In leaf, 16 TaHMs genes were regulated by AC, and 15 TaHMs were highly expressed in AC group (Fig. 5b). These up- or down-regulated TaHMs were speculated to play important roles in AC-promoted wheat seedlings growth.

In addition to their important functions in growth, $H M s$ genes also play essential functions in plant defenses $[5,10,15,39]$. Here, information on TaHMs-mediated stress responses were discussed (Fig. 6-7 and Table 1). There were 86 TaHMTs to be differently expressed after HS, DS or HD treatment (Fig. 6a), and 45 TaHDMs, 20 TaHATs and 27 TaHDACs were induced by these stress treatments (Fig. 6b-d). In respond to SS, almost all of TaHMs, especially TaSDGs in cluster 3 (Fig. 7a), TaJMJs in cluster 4 (Fig. 7b), TaHATs in cluster 2 (Fig. 7c), and TaHDACs in cluster 3 (Fig. 7d), were induced. The expression patterns of ten TaHMs, including TaSDG73, TaSDG81, TaSDG89, TaJMJ7, TaJMJ11, TaJMJ40, TaJMJ42, TaHDA17, TaHDA20 and TaHDA22, were affected by S. avenae or S. graminum feeding (Table 1). N stress regulated four TaHMs (TaSDG73, TaJMJ3, TaJMJ11 and TaHDA20) genes expression (Table 1). Transcriptions of $13 \mathrm{TaHMs}$ genes were influenced by Cd treatment, and most of them were induced (Table 1). Several ZmHMs were up-regulated or down-regulated by drought (Table 2). Numbers of stressrelated elements were identified in TaHMs and $Z m H M s$, which can partly explain their responses to adversities. The above findings indicated methylation is occurring, when wheat and maize suffers to biotic or abiotic stresses. 
The multiple functions of TaHMs were discussed in Fig. 8 and Table S5. A total of 85 TaHMs were simultaneously regulated by two signals; Three treatments commonly controlled about $25 \mathrm{TaHMS}$; Nine TaHMs were identified to be up-regulated or down-regulated by four signals; And only one wheat gene was commonly regulated by five treatments. Diverse functions of these TaHMs indicated they are commonly essential for wheat growth and stress adaptions, and we should pay more attention for them in future. Moreover, all $Z m H M s$, except for $Z m J M J 2$ and $Z m J M J 4$, responding to drought stresses were also identified in developing seeds, indicating their roles in maize growth and stress adaptions.

\section{Conclusions}

TaHMs, HvHMs, SbHMs, SvHMs, SiHMs and ZmHMs were systematically identified in our study. Their bioinformation, including chromosome locations, protein structures, phylogenetic relationships, gene duplications, promoter analysis and gene structure, were characterized. Potential roles of TaHMs in developing grain, BR-mediated root growth as well as AC-regulated seedlings development, and functions of $Z m H M s$ in seed development as well as GA-mediated leaf growth were investigated using published RNA sequence data. Candidate wheat $H M s$ genes taking part in heat, drought, salt, insect feeding, nutrition as well as heavy metal stresses, and $Z m H M s$ involving in drought response were then analyzed. In summary, identification of $H M s$ genes in six gramineae species, and characterization of their expression profiles in wheat and maize will be helpful for future study.

\section{Methods}

\section{Identification and naming of $H M s$}

The HMM files of each type of HMs genes were downloaded from Pfam database (http://pfam.sanger.ac.uk/), according to published IDs (HMTs: SDG-PF00856, PRMT-PF05185; HDMs: HDMA-PF04433, JMJ-PF02373; HATs: HAG-PF00583, HAM-PF01853, HAC-PF08214, HAFPF09247; HDACs: HDA-PF00850, SRT-PF02146) [3]. Using HMMER 3.0 software, T. aestivum, H. vulgare, S. bicolor, S. viridis, S. italic and Z. mays genomes were searched with HMM files [43]. No available HDTs was found in Pfam database, we then obtained Arabidopsis and rice HDTs proteins [4] to blast above gramineae protein databases using 'Blast Several Sequence to a Big Database' tool of TBtools software [44]. Phylogenetic analyses were used to confirm putative gramineae HMs protein sequences. Based on their gene location on chromosome, they were named as described in previous studies [44-49].

\section{Protein domain compositions, gene structures, cis-acting elements in promoters, phylogenetic, orthologous genes and heatmap analyses}

Using protein sequences, protein domain files were generated from Batch CD-Search database (https://www.ncbi.nlm.nih.gov/Structure/bwrpsb/bwrpsb.cgi). Visualization of conserved motifs was completed with 'Visualize NCBI CDD Domain Pattern' of TBtools [44]. 'Gene Structure View (Advanced)' of TBtools [44] was employed to investigate HMs gene structures. Promoter analysis was performed in 
PlantCARE database (http://bioinformatics.psb.ugent.be/webtools/plantcare/html/), and visualized using 'Simple Biosequence Viewer' of TBtools [44]. MEGA X was used to construct phylogenetic tree with Maximum Likelihood method, and proteins sequence alignment was completed with ClustalW method [50]. Synteny blocks were obtained from genome sequence and general feature format version 3 (gff3). 'Fasta stats, Table Row Extract Or Filter, File Merge For Mcscanx, File Transformat For Microsyteny Viewer And Advanced Circos' tools of TBtools [44] were used to visualize synteny relationships of homologous HMs genes. Using 'Simple Ka/Ks Calculator (NG)' of TBtools, the non-synonymous (Ka) and synonymous (Ks) nucleotide substitutions of orthologous gene pairs were calculated with coding sequeces [44]. The heat maps were generated with 'HeatMap' tool of TBtools [44].

\section{Plant materials and treatments}

Seed development After verbalization at $8^{\circ} \mathrm{C}$ for 8 weeks, bread wheat cv. Holdfast seedlings were transplanted to pots containing substrate and slow-release fertilizer. In glasshouse, plants were grown under follow conditions: $16 \mathrm{~h}$ light $\left(18^{\circ} \mathrm{C}\right), 8 \mathrm{~h}$ dark $\left(14^{\circ} \mathrm{C}\right)$. Wheat immature grain were dissected into endosperm, inner and outer seed pericarp tissues at 12 days postanthesis, and sampled for library construction and RNA sequencing (RNA-seq) [51]. Fragments Per Kilobase per Million (FPKM) values in above tissues were downloaded from wheat expression database (https://wheat.pw.usda.gov/WheatExp/about.html). In 2018, regular maize B73 grew in field, seeds were sampled at each growth phase, and waxy maize inbred line SWL01 was grown and sampled as the same way as B73; And we downloaded transcriptome data from previous study [35].

BR treatment Before cultured in aqueous solution under $22^{\circ} \mathrm{C}$ and a long-day ( $16 \mathrm{~h}$ light, $8 \mathrm{~h}$ dark) condition, seeds of common wheat ( $T$. aestivum $L$.) were sterilized and vernalized. Wheat seedlings (3day-old) treated with $50 \mathrm{nM}$ epibrassinolide (EpiBL, Sigma) as BR1 treated group, $1 \mathrm{mM}$ EpiBL as BR2 treated group and $1 \mathrm{mM}$ BRZ (BR synthesis inhibitor, Sigma) as BRZ treated group. After $12 \mathrm{~d}$ treatment, wheat roots were sampled and used for RNA-seq analysis [26]. Differentially expressed genes (DEGs) were provided by the corresponding author of published research [26].

$\mathrm{GA}_{3}$ treatment Dwarf $D 11$ is a GA-sensitive maize mutant. After sterilized with $2 \% \mathrm{NaClO}$ for $15 \mathrm{~min}, \mathrm{D} 11$ seeds were rinsed with distilled water and placed on filter paper. After germination, seeds were transferred to roseite. Seedlings treated with distilled water and $10^{-4} \mathrm{M} \mathrm{GA}_{3}$ (Sigma) every day were respectively recognized as control and GA treated group. The second leaf sheaths of $D 11$ were took at three fullyexpanded-leaves (V3) stage for RNA-seq, as previously described [36]. RNA-seq data were obtained from additional files of published study [36].

AC treatment After sterilization, immature embryos of wheat ( $T$. aestivum) were used to investigate the effect of AC (a wildly used growth regulator in plant tissue culture) on wheat seedlings growth in medium. Root $(\mathrm{R})$ and leaf $(\mathrm{L})$ were respectively used for RNA isolation and library construction. List of DEGs in published paper [30] was obtained from supplementary data. 
Heat, drought and salt treatments Heat and drought stress treatments are performed on wheat cultivar TAM 107 which is famous for heat and drought tolerant [31]. In control group, 7-day-old TAM 107 seedlings were growth in hydroponic solution under $16 \mathrm{~h}$ day $\left(22^{\circ} \mathrm{C}\right)$ and $8 \mathrm{~h}$ night $\left(18^{\circ} \mathrm{C}\right)$. Seedlings were treated with $40^{\circ} \mathrm{C}$ in heat stress (HS) group, seedlings were treated with $20 \%(\mathrm{~m} / \mathrm{V})$ PEG-6000 in drought stress (DS) group, and in HD group, seedlings were treated with combined heat $\left(40{ }^{\circ} \mathrm{C}\right)$ and drought stresses ( $40^{\circ} \mathrm{C}$ and $20 \%$ PEG-6000) [31]. Seedlings leaves were respectively sampled for RNA-seq after 1 and $6 \mathrm{~h}$ stress treatments. DEGs analysis was performed by Liu et al (2015) [31], and DEGs were downloaded from WheatExp database (https://wheat.pw.usda.gov/WheatExp/about.html). In terms of drought treatment for maize, drought sensitive ZX978 and tolerant ND476 were used in early study; Maize seedlings planted in soil with $70-80 \%$ and $15-20 \%$ water content were respectively set as control and drought treatment groups; At 12 day after drought treatment, flag leaves of ND476 and ZX978 under both control and drought treatment conditions were sampled for RNA-seq [52]. The seedlings of maize C7-2 which grew in growth chamber with $27^{\circ} \mathrm{C}, 60 \%$ humidity and $14 \mathrm{~h}$ day $/ 10 \mathrm{~h}$ night were also subjected to water stress; At 7 days after drought treatment, expanded third leaves of seven-day-old seedlings were sampled for RNA-seq analysis; And the DEGs detected between control and drought treated groups were got in additional file 2 of published data [53]. Two maize inbred lines (drought-sensitive inbred line Lv28 and drought-tolerant inbred line H082183) were also used for maize drought tolerance analysis; In control, moderate drought (MD) and severe drought (SD) treatment groups, seedlings were respectively well-watered, subjected to drought treatment of 27 and 46 days; Roots of Lv28 and H082183 were respectively sampled for RNA-seq, as described in Zhang et al. (2017) [54]. DEGs between drought (moderate and severe drought) and control group were referred to Zhang et al. (2017) [54].

Salt tolerance cultivar Qingmai 6 (QM) and sensitive cultivar Chinese Spring (CS) were used to detect response of wheat to salt [55]. Growth conditions of QM and CS seedlings was as the same as TAM 107. For salt treatment, $150 \mathrm{mmol} \mathrm{L}^{-1} \mathrm{NaCl}$ was added to solution. Roots of QM and CS were respectively collected at 6, 12, 24 and $48 \mathrm{~h}$ after salt treatment [55]. The salt related DEGs were identified in previous study [55].

Insect feeding, $\mathbf{N}$ and $\mathbf{C d}$ treatments In order to germinate seeds of Triticum aestivum var. Zhongmai 175 , they were cultured in distilled water for $72-96 \mathrm{~h}$ at $25^{\circ} \mathrm{C}$. Two-leaf stage wheat seedlings were used for adult aphid (non-phytotoxic aphid Sitobion avenae (S. avenae) and phytotoxic aphid Schizaphis graminum (S. graminum)) infestation [33]. Leaf samples of about $2.5 \times 2.5 \mathrm{~cm}$ from aphid feeding sites were used for RNA-sEq. Information on DEGs between control and treated samples were obtained from additional file 2 of published paper [33].

Uniformly wheat cultivar Wanmai No. 52 seedlings grow in hoagland nutrient solution under $12 \mathrm{~h}$ day $\left(20^{\circ} \mathrm{C}\right)$ and $12 \mathrm{~h}$ night $\left(15^{\circ} \mathrm{C}\right)$ condition. Two-leaf stage Wanmai No. 52 seedlings were used for $\mathrm{N}$ stress treatment, and roots and leaves were sampled for transcriptome analysis at ten days after treatment [56]. We downloaded DEGs in response to $\mathrm{N}$ stress from previous study [56]. 
Seedlings of $T$. aestivum L., cv Zhengmai 379 were grown in full nutrient solution under greenhouse with $8 \mathrm{~h}$ light $\left(187 \mu \mathrm{mol}\right.$ photon $\left.\mathrm{m}^{-2} \mathrm{~s}^{-1}, 23^{\circ} \mathrm{C}\right)$ and $16 \mathrm{~h}$ dark $\left(20^{\circ} \mathrm{C}\right)$. For cadmium $(\mathrm{Cd})$ stress treatment, $50 \mu \mathrm{M} \mathrm{CdCl} 2$ were applied to Zhengmai 379 seedlings. The roots of seedlings were harvested at 12 days after $\mathrm{Cd}$ treatment and used for transcriptome sequencing [34]. The DEGs identified in this study were found at its supplementary material [34].

For the delayed T. aestivum genome from International Wheat Genome Sequencing Consortium (IWGSC) and various maize genome versions, we converted gene ids of above wheat genes in the website (http://202.194.139.32/idConvert/) and maize gene ids in MaizeGDB database (https://chinese.maizegdb.org/gene_center/gene).

All plant materials mentioned here were used in previous studies, and the original source and the formal identification of the plant materials can be found.

\section{Abbreviations}

HATs: Histone acetylases; HDACs: Histone deacetylases; HDMs: Histone methylases; HM: Histone modification; HMTs: Histone methyltransferases; FPKM: Fragments Per Kilobase per Million; HS: heat stress; DS: drought stress; MD: moderate drought; SD: severe drought; S. avenae. Sitobion avenae; $S$. graminum: Schizaphis graminum; IWGSC: International Wheat Genome Sequencing Consortium.

\section{Declarations}

\section{Acknowledgments}

Thank for Pro. Shengbao Xu and Peng Zhao, Pro. Shuo Zhou and Fushuang Dong, and Pro. Jinxia Qin sharing transcriptome data.

\section{Authors' contributions}

JH conceived and designed the experiment. LZ, SM, DS, YW, HF and CY performed the experiments; LZ drafted the manuscript and analyzed the data; $\mathrm{JH}$ revised the final version of the manuscript. All authors read and approved the final manuscript.

\section{Funding}

This work was supported by Major collaborative innovation project of Zhengzhou (Key discipline construction project of Zhengzhou University) (xkzdjc201905). The funding agencies provided only financing for the experimental costs and publication fee for this work. Funds were used for the design of the study; the collection, analysis and interpretation of data; writing the manuscript and open access payment. 


\section{Availability of data and materials}

The datasets used and/or analysed during the current study are available from the corresponding author on reasonable request.

\section{Ethics approval and consent to participate}

Not applicable.

\section{Consent for publication}

Not applicable]

\section{Competing interests}

The authors declare there are no conflicts of interest.

\section{References}

1. Zhang X. The epigenetic landscape of plants. Science. 2008; 320(5875):489-492.

2. Klose RJ, Zhang Y. Regulation of histone methylation by demethylimination and demethylation. Nat Rev Mol Cell Biol. 2007; 8(4):307-318.

3. Xu J, Xu H, Liu Y, Wang X, Xu Q, Deng X. Genome-wide identification of sweet orange (Citrus sinensis) histone modification gene families and their expression analysis during the fruit development and fruit-blue mold infection process. Frontiers in Plant Science. 2015; 6:607.

4. Aiese CR, Sanseverino W, Cremona G, Ercolano MR, Conicella C, Consiglio FM. Genome-wide analysis of histone modifiers in tomato: gaining an insight into their developmental roles. Bmc Genomics. 2013; 14:57.

5. Ahmad A, Cao X. Plant PRMTs broaden the scope of arginine methylation. Journal of Genetics and Genomics. 2012; 39(5):195-208.

6. Dong G, Ma DP, Li J. The histone methyltransferase SDG8 regulates shoot branching in Arabidopsis. Biochem Biophys Res Commun. 2008; 373(4):659-664.

7. Jiang D, Yang W, He Y, Amasino RM. Arabidopsis relatives of the human lysine-specific Demethylase 1 repress the expression of FWA and FLOWERING LOCUS C and thus promote the floral transition. Plant Cell. 2007; 19(10):2975-2987.

8. Chen $X$, Hu Y, Zhou DX. Epigenetic gene regulation by plant Jumonji group of histone demethylase. Biochim Biophys Acta. 2011; 1809(8):421-426.

9. Pandey R, Muller A, Napoli CA, Selinger DA, Pikaard CS, Richards EJ, Bender J, Mount DW, Jorgensen RA. Analysis of histone acetyltransferase and histone deacetylase families of Arabidopsis thaliana 
suggests functional diversification of chromatin modification among multicellular eukaryotes. Nucleic Acids Research. 2002; 30(23):5036-5055.

10. Tian L, Fong MP, Wang JJ, Wei NE, Jiang H, Doerge RW, Chen ZJ. Reversible histone acetylation and deacetylation mediate genome-wide, promoter-dependent and locus-specific changes in gene expression during plant development. Genetics. 2005; 169(1):337-345.

11. Sheldon CC, Finnegan EJ, Dennis ES, Peacock WJ. Quantitative effects of vernalization on FLC and SOC1 expression. Plant Journal. 2006; 45(6):871-883.

12. Chen ZJ, Tian L. Roles of dynamic and reversible histone acetylation in plant development and polyploidy. Biochim Biophys Acta. 2007; 1769(5-6):295-307.

13. Hollender C, Liu Z. Histone deacetylase genes in Arabidopsis development. Journal of Integrative Plant Biology. 2008; 50(7):875-885.

14. Ma X, Lv S, Zhang C, Yang C. Histone deacetylases and their functions in plants. Plant Cell Reports. 2013; 32(4):465-478.

15. Wang Z, Cao H, Chen F, Liu Y. The roles of histone acetylation in seed performance and plant development. Plant Physiol Biochem. 2014; 84:125-133.

16. Appels R, Eversole K, Feuillet C, Keller B, Rogers J, Stein N, Pozniak CJ, Stein N, Choulet F, Distelfeld A et al. Shifting the limits in wheat research and breeding using a fully annotated reference genome. Science. 2018; 361(6403).

17. Steele KA, Price AH, Witcombe JR, Shrestha R, Singh BN, Gibbons JM, Virk DS. QTLs associated with root traits increase yield in upland rice when transferred through marker-assisted selection. Theoretical and Applied Genetics. 2013; 126(1):101-108.

18. Uga Y, Sugimoto K, Ogawa S, Rane J, Ishitani M, Hara N, Kitomi Y, Inukai Y, Ono K, Kanno N et al. Control of root system architecture by DEEPER ROOTING 1 increases rice yield under drought conditions. Nature Genetics. 2013; 45(9):1097-1102.

19. Zorb C, Geilfus CM, Dietz KJ. Salinity and crop yield. Plant Biol (Stuttg). 2019; 21 Suppl 1:31-38.

20. Hanin M, Ebel C, Ngom M, Laplaze L, Masmoudi K. New Insights on Plant Salt Tolerance Mechanisms and Their Potential Use for Breeding. Frontiers in Plant Science. 2016; 7:1787.

21. Xie W, Xiong W, Pan J, Ali T, Cui Q, Guan D, Meng J, Mueller ND, Lin E, Davis SJ. Decreases in global beer supply due to extreme drought and heat. Nature Plants. 2018; 4(11):964-973.

22. Wang X, Xin C, Cai J, Zhou Q, Dai T, Cao W, Jiang D. Heat Priming Induces Trans-generational Tolerance to High Temperature Stress in Wheat. Frontiers in Plant Science. 2016; 7:501.

23. Chen D, Chen D, Xue R, Long J, Lin X, Lin Y, Jia L, Zeng R, Song Y. Effects of boron, silicon and their interactions on cadmium accumulation and toxicity in rice plants. Journal of Hazardous Materials. $2019 ; 367: 447-455$.

24. Chinnusamy V, Zhu JK. Epigenetic regulation of stress responses in plants. Current Opinion in Plant Biology. 2009; 12(2):133-139. 
25. Choi SM, Song HR, Han SK, Han M, Kim CY, Park J, Lee YH, Jeon JS, Noh YS, Noh B. HDA19 is required for the repression of salicylic acid biosynthesis and salicylic acid-mediated defense responses in Arabidopsis. Plant Journal. 2012; 71(1):135-146.

26. Hou L, Zhang A, Wang R, Zhao P, Zhang D, Jiang Y, Diddugodage CJ, Wang X, Ni Z, Xu S. Brassinosteroid Regulates Root Development with Highly Redundant Genes in Hexaploid Wheat. Plant and Cell Physiology. 2019; 60(8):1761-1777.

27. Konishi H, Komatsu S. A proteomics approach to investigating promotive effects of brassinolide on lamina inclination and root growth in rice seedlings. Biological \& Pharmaceutical Bulletin. 2003; 26(4):401-408.

28. Wei Z, Li J. Brassinosteroids Regulate Root Growth, Development, and Symbiosis. Molecular Plant. 2016; $9(1): 86-100$.

29. Kim SK, Chang SC, Lee EJ, Chung WS, Kim YS, Hwang S, Lee JS. Involvement of brassinosteroids in the gravitropic response of primary root of maize. Plant Physiology. 2000; 123(3):997-1004.

30. Dong FS, Lv MY, Wang JP, Shi XP, Liang XX, Liu YW, Yang F, Zhao H, Chai JF, Zhou S. Transcriptome analysis of activated charcoal-induced growth promotion of wheat seedlings in tissue culture. Bmc Genetics. 2020; 21(1):69.

31. Liu Z, Xin M, Qin J, Peng H, Ni Z, Yao Y, Sun Q. Temporal transcriptome profiling reveals expression partitioning of homeologous genes contributing to heat and drought acclimation in wheat (Triticum aestivum L.). Bmc Plant Biology. 2015; 15:152.

32. Hu XS, Liu XF, Thieme T, Zhang GS, Liu TX, Zhao HY. Testing the fecundity advantage hypothesis with Sitobion avenae, Rhopalosiphum padi, and Schizaphis graminum (Hemiptera: Aphididae) feeding on ten wheat accessions. Sci Rep. 2015; 5:18549.

33. Zhang Y, Fu Y, Fan J, Li Q, Francis F, Chen J. Comparative transcriptome and histological analyses of wheat in response to phytotoxic aphid Schizaphis graminum and non-phytotoxic aphid Sitobion avenae feeding. Bmc Plant Biology. 2019; 19(1):547.

34. Qin S, Liu H, Rengel Z, Gao W, Nie Z, Li C, Hou M, Cheng J, Zhao P. Boron inhibits cadmium uptake in wheat (Triticum aestivum) by regulating gene expression. Plant Science. 2020; 297:110522.

35. Gu W, Yu D, Guan Y, Wang H, Qin T, Sun P, Hu Y, Wei J, Zheng H. The dynamic transcriptome of waxy maize (Zea mays L. sinensis Kulesh) during seed development. Genes Genomics. 2020; 42(9):9971010.

36. Wang Y, Wang X, Deng D, Wang Y. Maize transcriptomic repertoires respond to gibberellin stimulation. Molecular Biology Reports. 2019; 46(4):4409-4421.

37. Lu F, Li G, Cui X, Liu C, Wang XJ, Cao X. Comparative analysis of JmjC domain-containing proteins reveals the potential histone demethylases in Arabidopsis and rice. Journal of Integrative Plant Biology. 2008; 50(7):886-896.

38. Xu G, Guo C, Shan H, Kong H. Divergence of duplicate genes in exon-intron structure. Proc Natl Acad Sci U S A. 2012; 109(4):1187-1192. 
39. Berr A, McCallum EJ, Alioua A, Heintz D, Heitz T, Shen WH. Arabidopsis histone methyltransferase SET DOMAIN GROUP8 mediates induction of the jasmonate/ethylene pathway genes in plant defense response to necrotrophic fungi. Plant Physiology. 2010; 154(3):1403-1414.

40. Kim JM, To TK, Nishioka T, Seki M. Chromatin regulation functions in plant abiotic stress responses. Plant Cell and Environment. 2010; 33(4):604-611.

41. Luo M, Liu X, Singh P, Cui Y, Zimmerli L, Wu K. Chromatin modifications and remodeling in plant abiotic stress responses. Biochim Biophys Acta. 2012; 1819(2):129-136.

42. Lynch M, Conery JS. The evolutionary fate and consequences of duplicate genes. Science. 2000; 290(5494):1151-1155.

43. Finn RD, Clements J, Eddy SR. HMMER web server: interactive sequence similarity searching. Nucleic Acids Research. 2011; 39(Web Server issue):W29-W37.

44. Chen C, Chen H, Zhang Y, Thomas HR, Frank MH, He Y, Xia R. TBtools: An Integrative Toolkit Developed for Interactive Analyses of Big Biological Data. Molecular Plant. 2020; 13(8):1194-1202.

45. Zheng L, Ma J, Song C, An N, Zhang D, Zhao C, Qi S, Han M. Genome-wide identification and expression profiling analysis of brassinolide signal transduction genes regulating apple tree architecture. Acta Physiologiae Plantarum. 2017; 39(8):177.

46. Zheng L, Ma J, Song C, Zhang L, Gao C, Zhang D, An N, Mao J, Han M. Genome-wide identification and expression analysis of GRF genes regulating apple tree architecture. Tree Genetics \& Genomes. 2018; 14(544).

47. Zheng L, Zhao C, Mao J, Song C, Ma J, Zhang D, Han M, An N. Genome-wide identification and expression analysis of brassinosteroid biosynthesis and metabolism genes regulating apple tree shoot and lateral root growth. Journal of Plant Physiology. 2018; 231:68-85.

48. Zheng L, Ma J, Zhang L, Gao C, Zhang D, Zhao C, Han M. Revealing critical mechanisms of BRmediated apple nursery tree growth using iTRAQ-based proteomic analysis. Journal of Proteomics. 2018; 173:139-154.

49. Zheng L, Yang Y, Gao C, Ma J, Shah K, Zhang D, Zhao C, Xing L, Han M, An N et al. Transcriptome Analysis Reveals New Insights into MdBAK1-Mediated Plant Growth in Malus domestica. J Agric Food Chem. 2019; 67(35):9757-9771.

50. Kumar S, Stecher G, Li M, Knyaz C, Tamura K. MEGA X: Molecular Evolutionary Genetics Analysis across Computing Platforms. Molecular Biology and Evolution. 2018; 35(6):1547-1549.

51. Wan Y, Underwood C, Toole G, Skeggs P, Zhu T, Leverington M, Griffiths S, Wheeler T, Gooding M, Poole R et al. A novel transcriptomic approach to identify candidate genes for grain quality traits in wheat. Plant Biotechnology Journal. 2009; 7(5):401-410.

52. Liu G, Zenda T, Liu S, Wang X, Jin H, Dong A, Yang Y, Duan H. Comparative transcriptomic and physiological analyses of contrasting hybrid cultivars ND476 and ZX978 identify important differentially expressed genes and pathways regulating drought stress tolerance in maize. Genes Genomics. 2020; 42(8):937-955. 
53. Zhang Q, Liu H, Wu X, Wang W. Identification of drought tolerant mechanisms in a drought-tolerant maize mutant based on physiological, biochemical and transcriptomic analyses. Bmc Plant Biology. 2020; 20(1):315.

54. Zhang X, Liu X, Zhang D, Tang H, Sun B, Li C, Hao L, Liu C, Li Y, Shi Y et al. Genome-wide identification of gene expression in contrasting maize inbred lines under field drought conditions reveals the significance of transcription factors in drought tolerance. PLoS One. 2017; 12(7):e179477.

55. Zhang Y, Liu Z, Khan AA, Lin Q, Han Y, Mu P, Liu Y, Zhang H, Li L, Meng X et al. Expression partitioning of homeologs and tandem duplications contribute to salt tolerance in wheat (Triticum aestivum L.). Sci Rep. 2016; 6:21476.

56. Wang J, Song K, Sun L, Qin Q, Sun Y, Pan J, Xue Y. Morphological and Transcriptome Analysis of Wheat Seedlings Response to Low Nitrogen Stress. Plants (Basel). 2019; 8(4).

\section{Tables}


Table 1

Expression analysis of TaHMs during different biotic and abiotic stresses

\begin{tabular}{|c|c|c|c|c|c|}
\hline Gene & $\mathrm{Sa} / \mathrm{C}$ & $\mathrm{Sg} / \mathrm{C}$ & N/C_leaf & N/C_root & $\mathrm{Cd} / \mathrm{C}$ \\
\hline TaJMJ7 & 3.460546762 & 4.061182894 & Nan & Nan & Nan \\
\hline TaJMJ11 & 4.25128801 & Nan & Nan & 3.242752801 & Nan \\
\hline TaJMJ40 & Nan & 176.8521564 & Nan & Nan & Nan \\
\hline TaJMJ42 & Nan & 12.76303914 & Nan & Nan & Nan \\
\hline TaHDA17 & Nan & 0.19218931 & Nan & Nan & Nan \\
\hline TaSDG73 & Nan & 0.095377977 & 0.12244898 & Nan & Nan \\
\hline TaHDA20 & Nan & 0.198773867 & 0.114219114 & Nan & Nan \\
\hline TaSDG81 & Nan & 0.109196612 & Nan & Nan & Nan \\
\hline TaHDA22 & Nan & 0.161925264 & Nan & Nan & Nan \\
\hline TaSDG89 & Nan & 0.06737233 & Nan & Nan & Nan \\
\hline TaJMJ3 & Nan & Nan & Nan & 3.687573184 & Nan \\
\hline TaSDG13 & Nan & Nan & Nan & Nan & 6.470609988 \\
\hline TaSDG100 & Nan & Nan & Nan & Nan & 4.039565068 \\
\hline TaSDG102 & Nan & Nan & Nan & Nan & 0.265806191 \\
\hline TaSDG66 & Nan & Nan & Nan & Nan & 4.300055702 \\
\hline TaSDG74 & Nan & Nan & Nan & Nan & 3.367466059 \\
\hline TaSDG112 & Nan & Nan & Nan & Nan & 3.127601118 \\
\hline TaSDG82 & Nan & Nan & Nan & Nan & 3.248304396 \\
\hline TaSDG106 & Nan & Nan & Nan & Nan & 2.966738703 \\
\hline TaSDG62 & Nan & Nan & Nan & Nan & 3.971222851 \\
\hline TaJMJ28 & Nan & Nan & Nan & Nan & 2.453364919 \\
\hline TaHDT1 & Nan & Nan & Nan & Nan & 2.228164872 \\
\hline TaJMJ34 & Nan & Nan & Nan & Nan & 2.353792646 \\
\hline TaSDG87 & Nan & Nan & Nan & Nan & 2.360930064 \\
\hline
\end{tabular}


Table 2

Expression analysis of $\mathrm{ZmHMs}$ during different drought stresses

\begin{tabular}{|c|c|c|c|c|c|c|}
\hline Gene_id & TD/SD & $\mathrm{CD} / \mathrm{CC}$ & LMD/LMC & LSD/LSC & HMD/HMC & HSD/HSD \\
\hline ZmJMJ2 & 6.086 & Nan & Nan & Nan & Nan & Nan \\
\hline$Z m H D A 11$ & 0.249 & Nan & Nan & Nan & Nan & Nan \\
\hline ZmSDG5 & Nan & 9.388824371 & Nan & Nan & Nan & Nan \\
\hline ZmJMJ4 & Nan & 3.074936123 & Nan & Nan & Nan & Nan \\
\hline$Z m S D G 24$ & Nan & 2.363578856 & Nan & Nan & Nan & Nan \\
\hline ZmSDG33 & Nan & 0.457540122 & Nan & Nan & Nan & Nan \\
\hline ZmJMJ17 & Nan & 0.390122323 & Nan & Nan & Nan & Nan \\
\hline ZmJMJ5 & Nan & Nan & Up & Nan & Nan & Up \\
\hline$Z m S D G 1$ & Nan & Nan & Nan & Nan & Up & Nan \\
\hline$Z m H D A 2$ & Nan & Nan & Nan & Nan & Nan & Down \\
\hline \multicolumn{7}{|c|}{$\begin{array}{l}\text { TD: tolerant cultivar ND476 drought treatment, SD: sensitive cultivar ZX978 drought treatment, CD: } \\
\text { C7-2 drought treatment, CC: C7-2 control, LMD: Lv28 moderate drought treatment, LMC: Lv28 } \\
\text { control, LSD: LV28 severe drought treatment, LSC: Lv28 control, HMD: H082183 moderate drought } \\
\text { treatment, HMC: H082183 control, HSD: H082183 severe drought treatment, HSC: H082183 control. }\end{array}$} \\
\hline
\end{tabular}

\section{Figures}



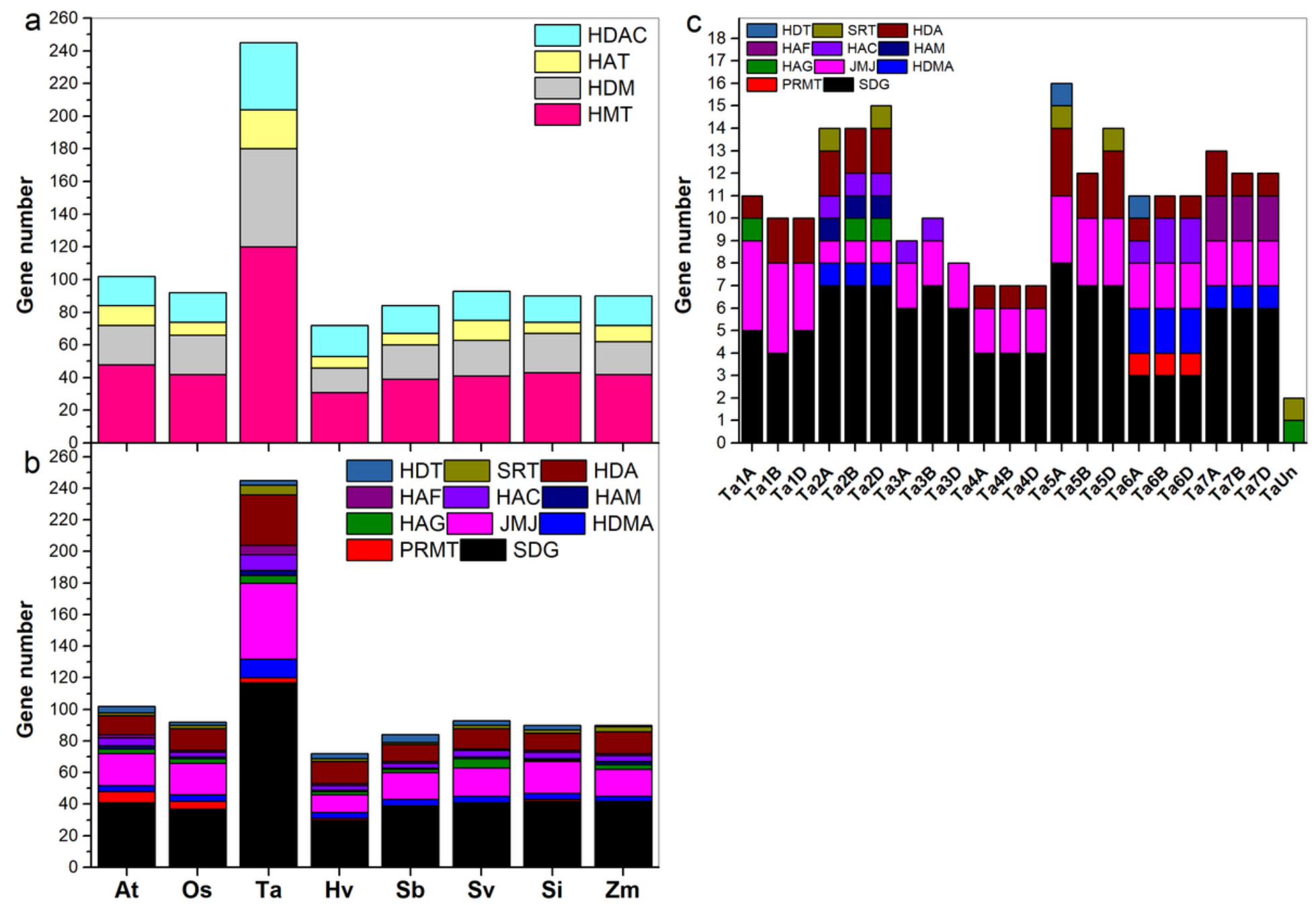

Figure 1

The number of HMs genes among different species. a Gene number of each HMs families in Arabidopsis, rice and six gramineaes. b Gene number of each HMs subfamilies in model and six gramineaes. $c$ The number of each TaHMs subfamilies on wheat chromosomes. 

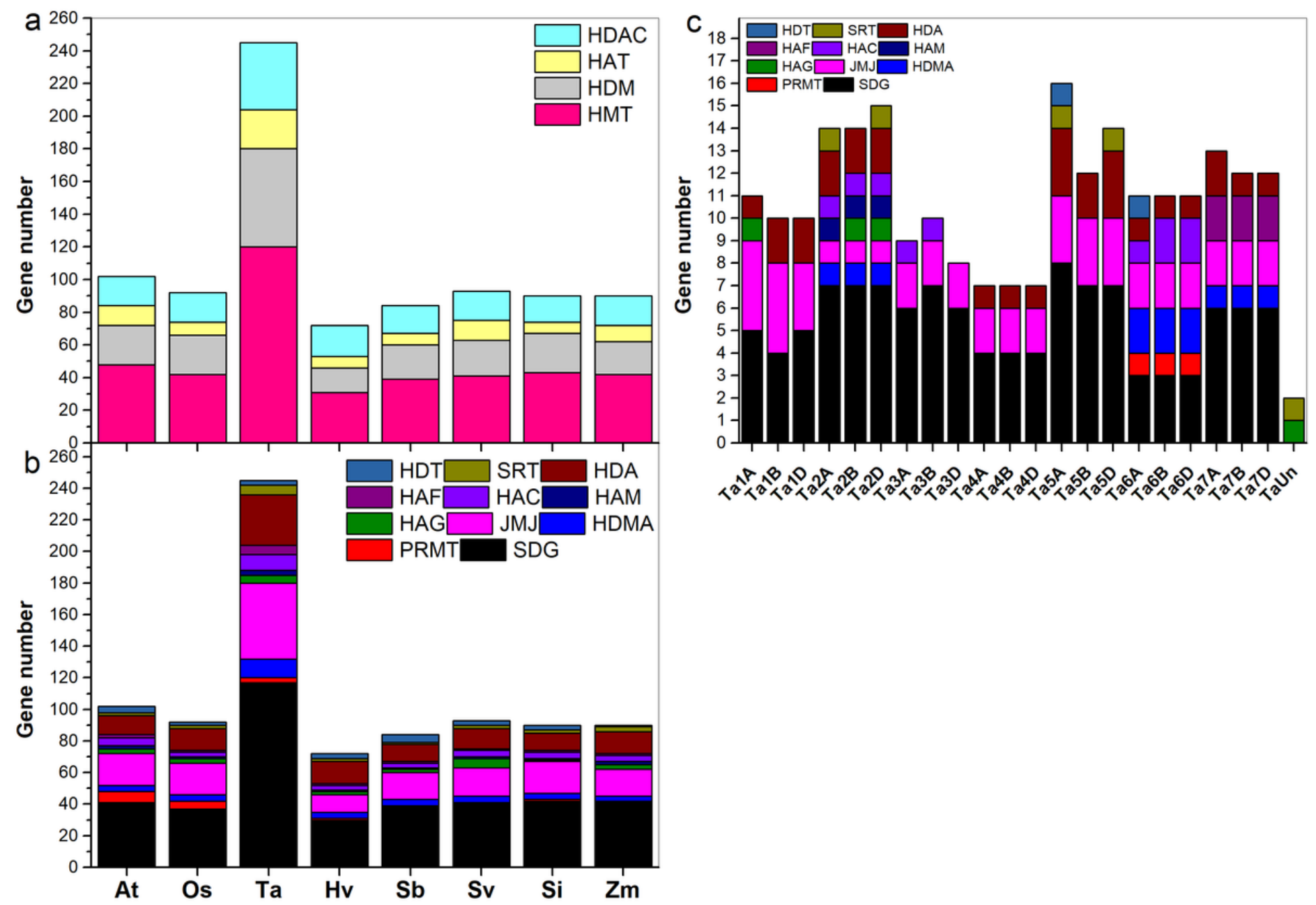

Figure 1

The number of HMs genes among different species. a Gene number of each HMs families in Arabidopsis, rice and six gramineaes. b Gene number of each HMs subfamilies in model and six gramineaes. $c$ The number of each TaHMs subfamilies on wheat chromosomes. 

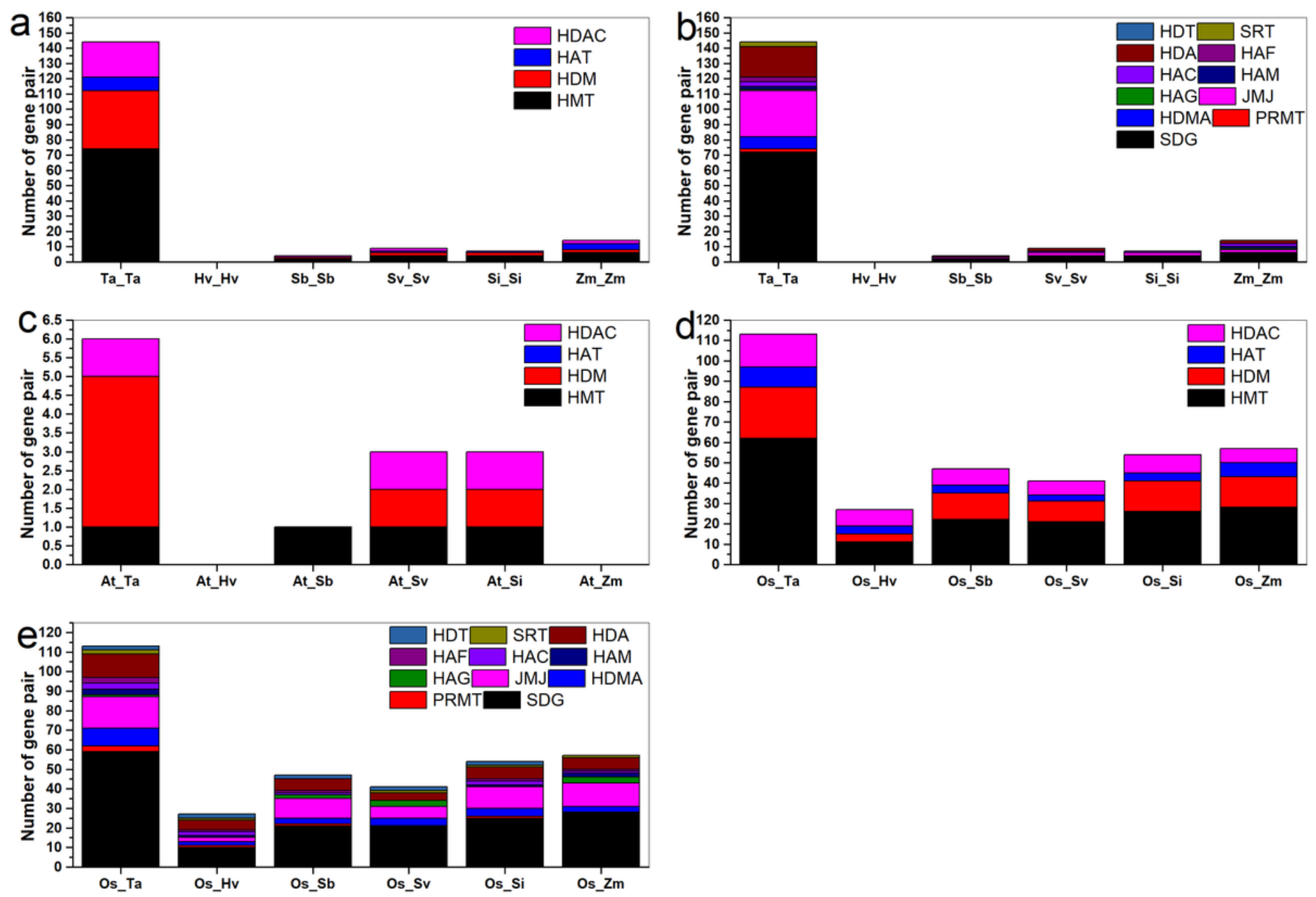

Figure 2

Comparison the number of genes orthology against with different genomes. a The number of each HMs family gene pair among each gramineae specie. $b$ The number of each HMs subfamily gene pair among each gramineae specie. c The number of each HMs gene pair between Arabidopsis and each gramineae specie. $d$ The number of each HMs family gene pair between rice and each gramineae specie. $e$ The number of each HMs subfamily gene pair between rice and each gramineae specie. 

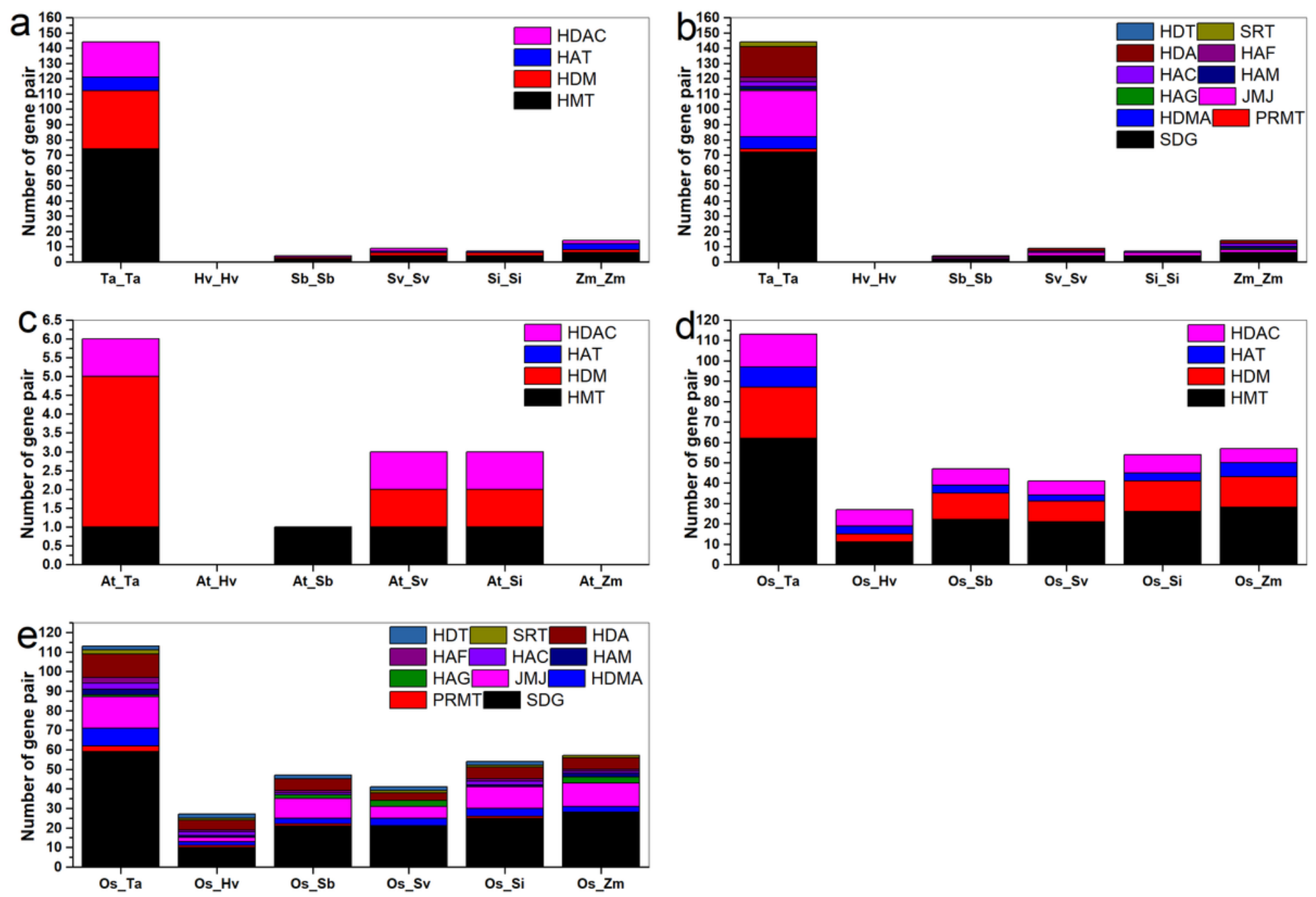

Figure 2

Comparison the number of genes orthology against with different genomes. a The number of each HMs family gene pair among each gramineae specie. $b$ The number of each HMs subfamily gene pair among each gramineae specie. c The number of each HMs gene pair between Arabidopsis and each gramineae specie. $d$ The number of each HMs family gene pair between rice and each gramineae specie. $e$ The number of each HMs subfamily gene pair between rice and each gramineae specie. 

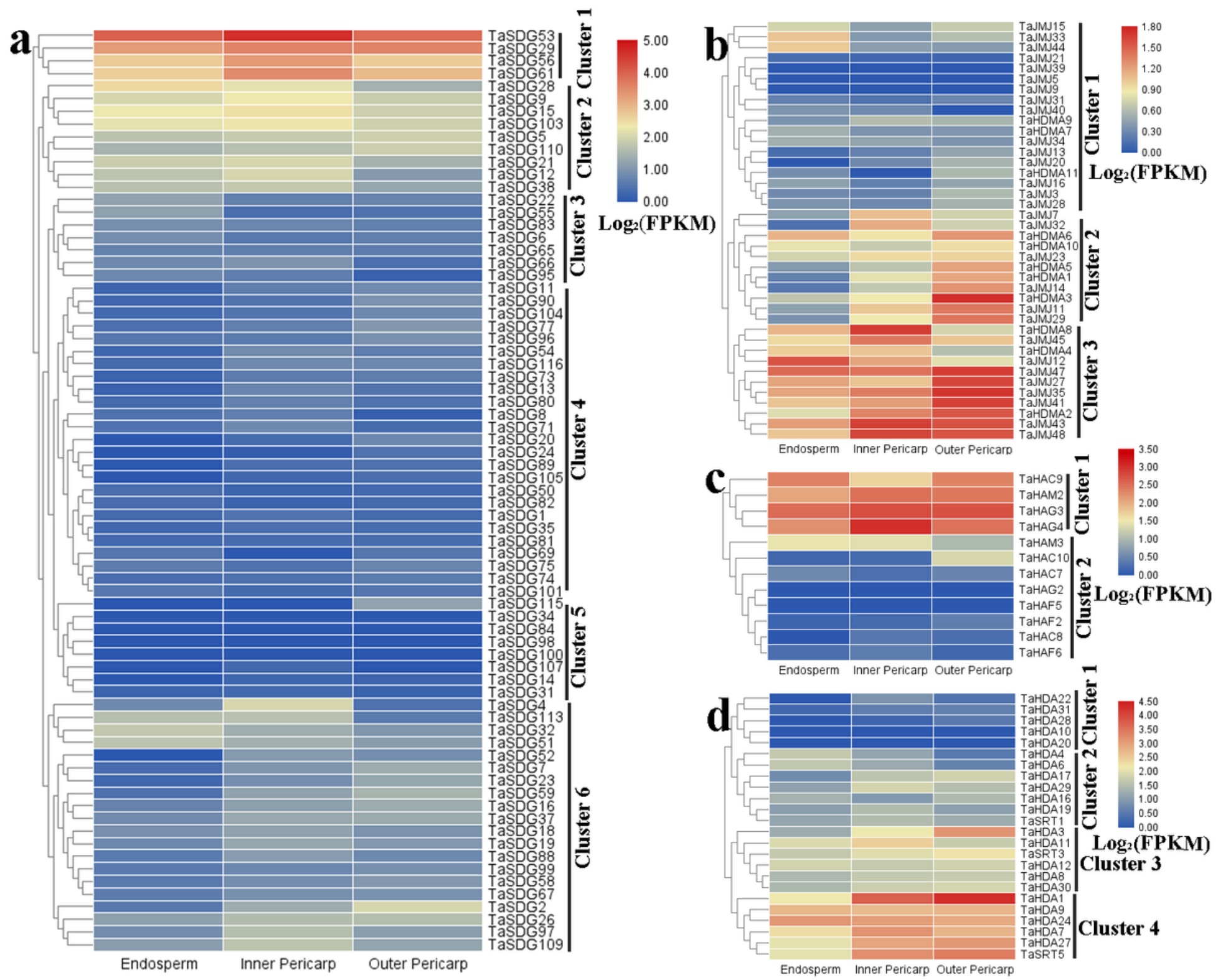

\section{Figure 3}

Expression profiles of TaHMs genes in various tissues of developing grain. a TaHMTs: TaSDGs. $b$ TaHDMs: TaHDMAs and TaJMJs. c TaHATs: TaHAGs, TaHAMs, TaHACs and TaHAFs. d TaHDACs: TaHDAs, TaSRTs and TaHDTs. FPKM: Fragments per kilobase per million. Expressions of the other TaHMs not shown here were not detected in developing grain. 

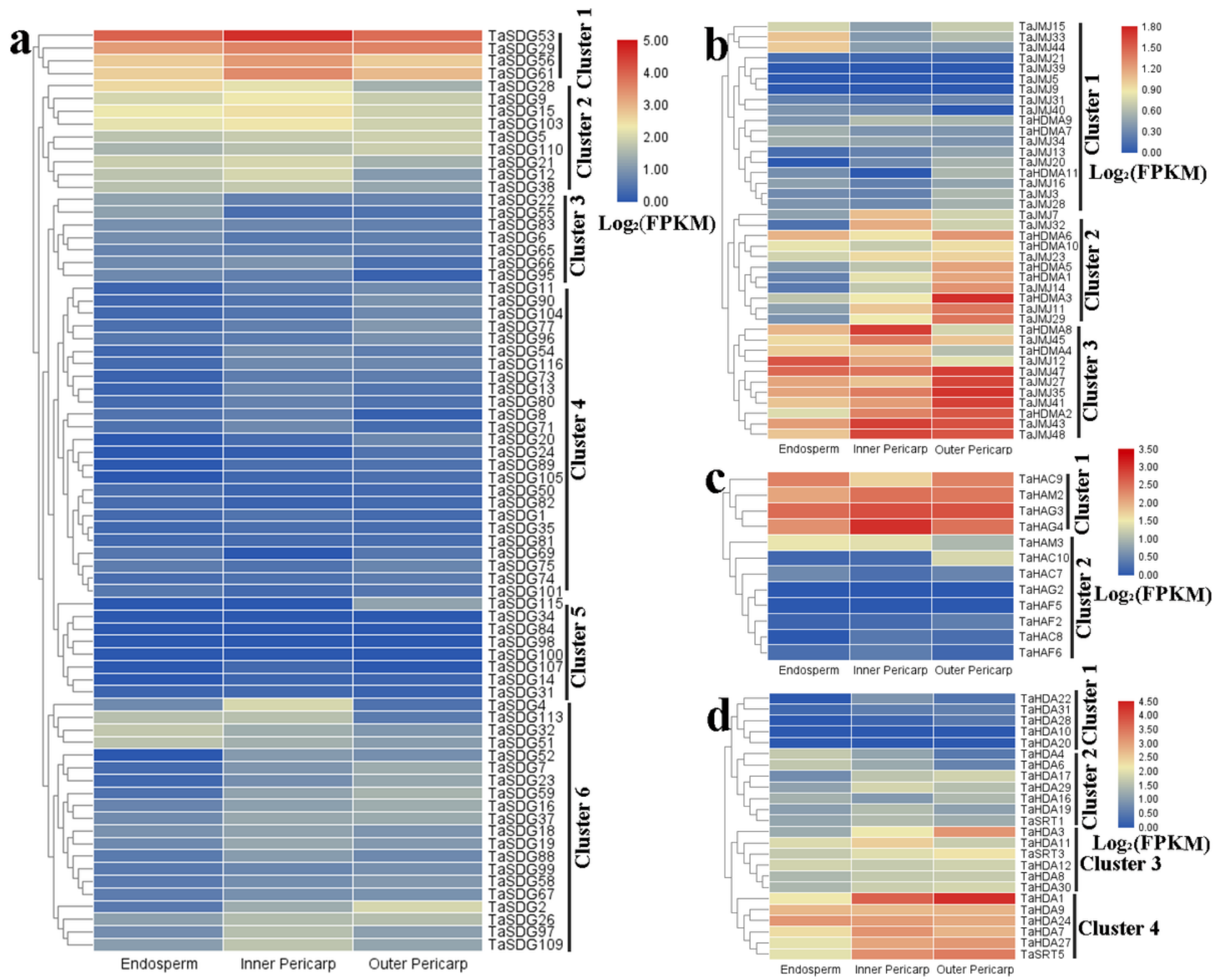

\section{Figure 3}

Expression profiles of TaHMs genes in various tissues of developing grain. a TaHMTs: TaSDGs. $b$ TaHDMs: TaHDMAs and TaJMJs. c TaHATs: TaHAGs, TaHAMs, TaHACs and TaHAFs. d TaHDACs: TaHDAs, TaSRTs and TaHDTs. FPKM: Fragments per kilobase per million. Expressions of the other TaHMs not shown here were not detected in developing grain. 

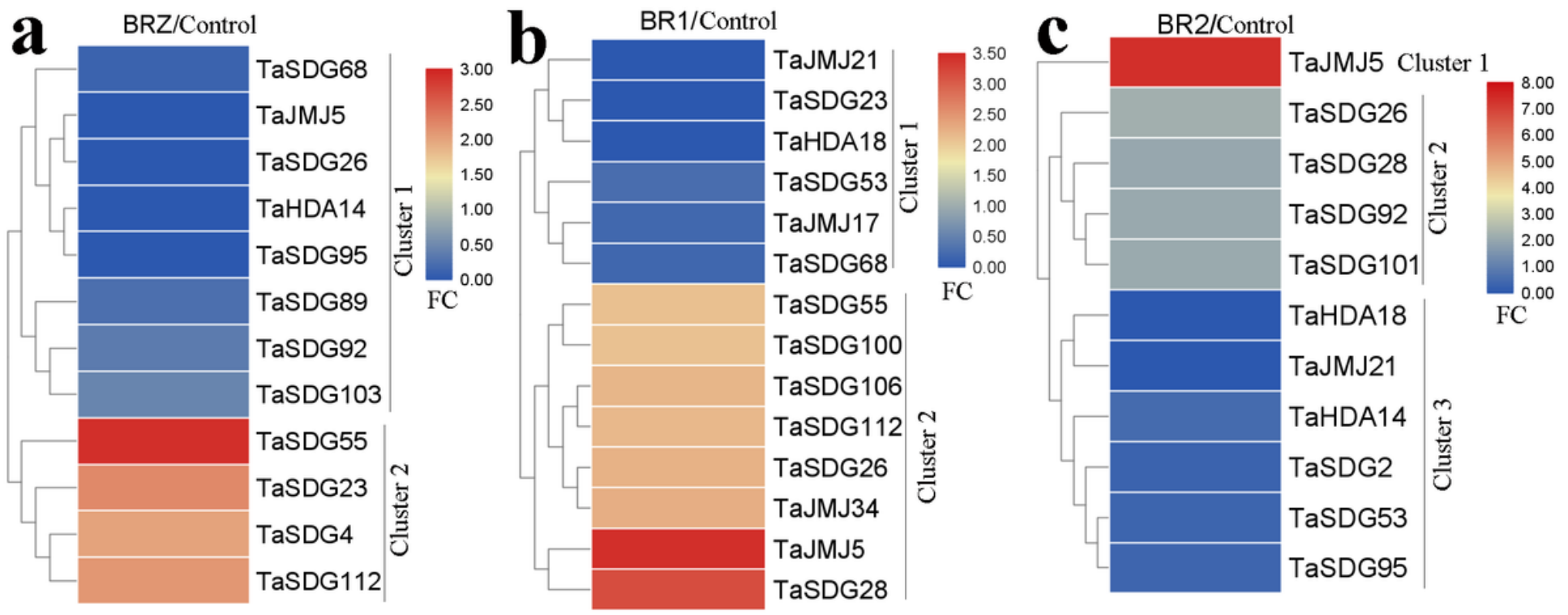

Figure 4

Expression analysis of TaHMs in response to BR and BRZ. a Differently expressed TaHMs between BRZtreated and control groups. b Differently expressed TaHMs between BR1-treated and control groups. C Differently expressed TaHMs between BR2-treated and control groups. BR1, 50 nM EpiBL; BR2, 1 mM EpiBL; BRZ, 1 mM BRZ. FC: fold change.
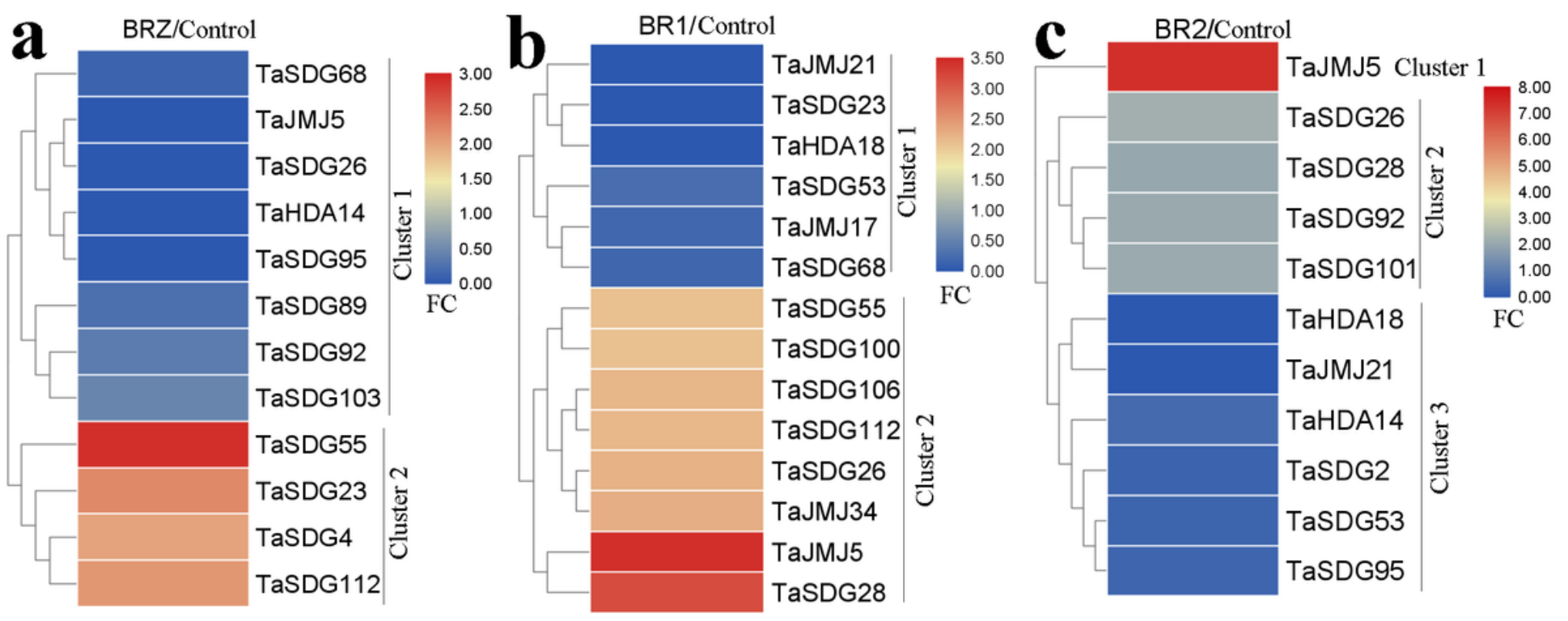

\section{Figure 4}

Expression analysis of TaHMs in response to BR and BRZ. a Differently expressed TaHMs between BRZtreated and control groups. b Differently expressed TaHMs between BR1-treated and control groups. C Differently expressed TaHMs between BR2-treated and control groups. BR1, $50 \mathrm{nM}$ EpiBL; BR2, $1 \mathrm{mM}$ EpiBL; BRZ, 1 mM BRZ. FC: fold change. 


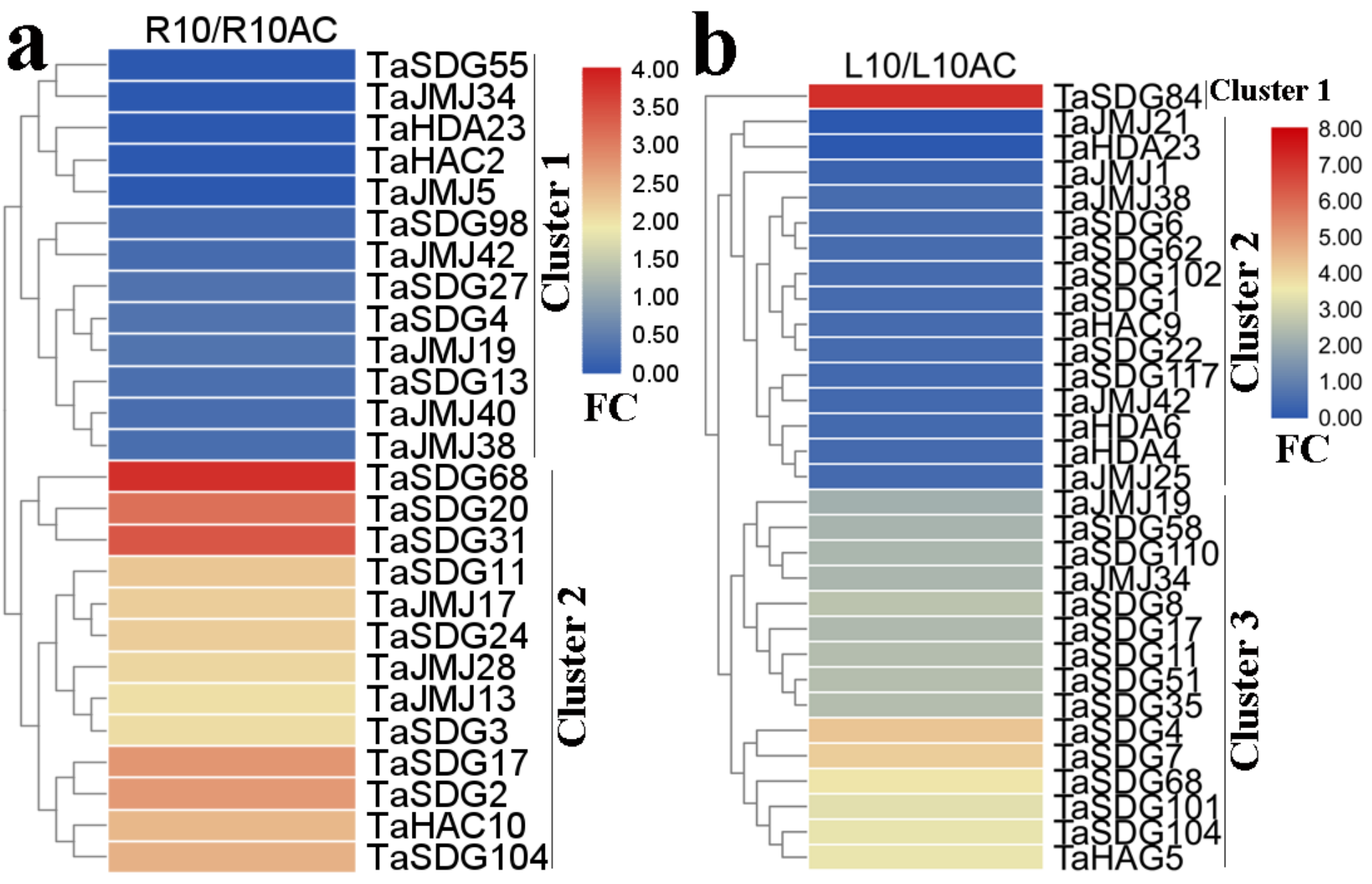

Figure 5

Expression pattern analysis of TaHMs in response to AC. a Differently expressed TaHMs between AC (R10AC) and control (R10) groups in root of 10-day-old seedlings. b Differently expressed TaHMs between AC (L10) and control (L10AC) groups in leaf of 10-day-old seedlings. FC: fold change. 


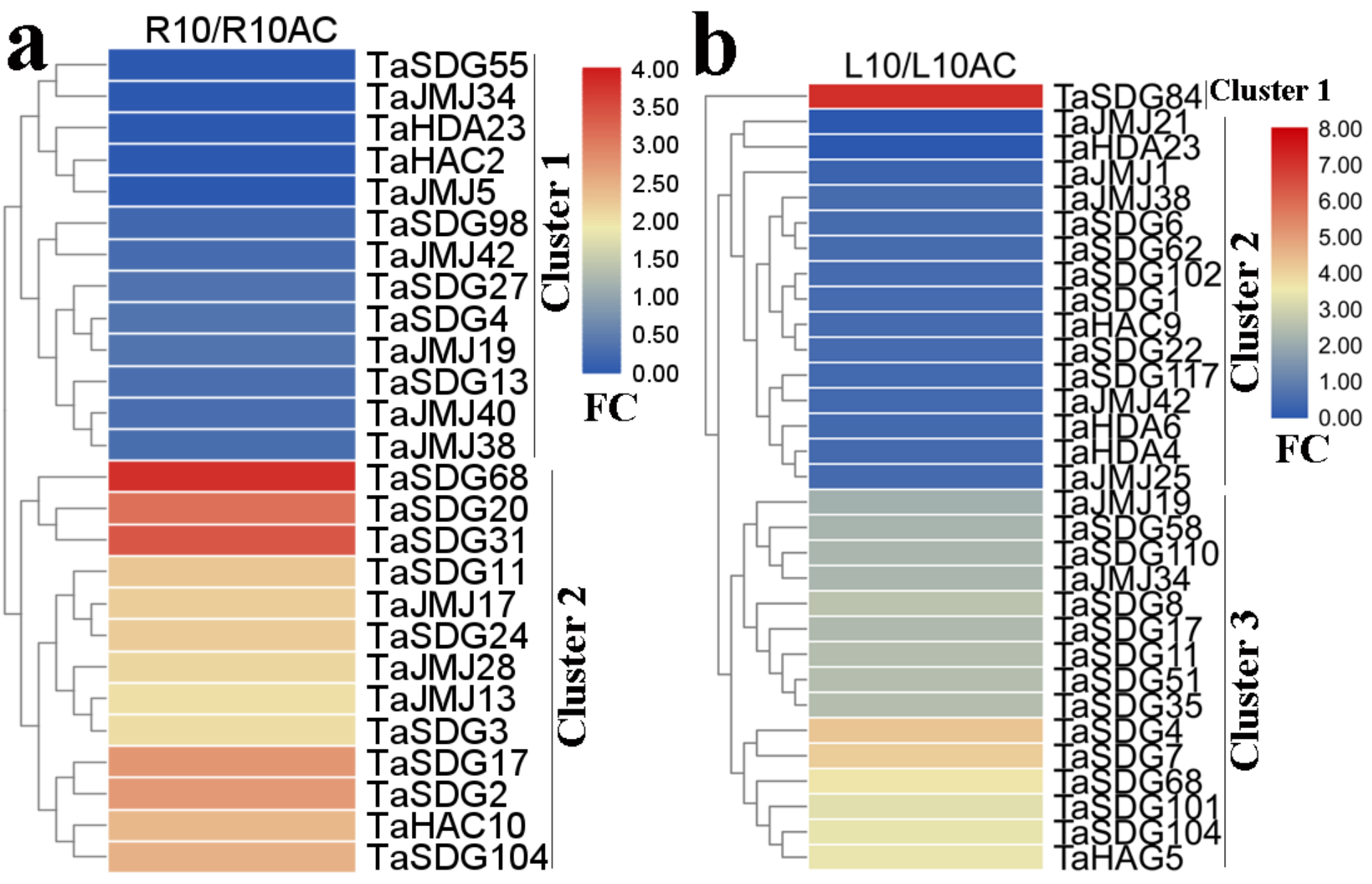

Figure 5

Expression pattern analysis of TaHMs in response to AC. a Differently expressed TaHMs between AC (R10AC) and control (R10) groups in root of 10-day-old seedlings. b Differently expressed TaHMs between AC (L10) and control (L10AC) groups in leaf of 10-day-old seedlings. FC: fold change. 

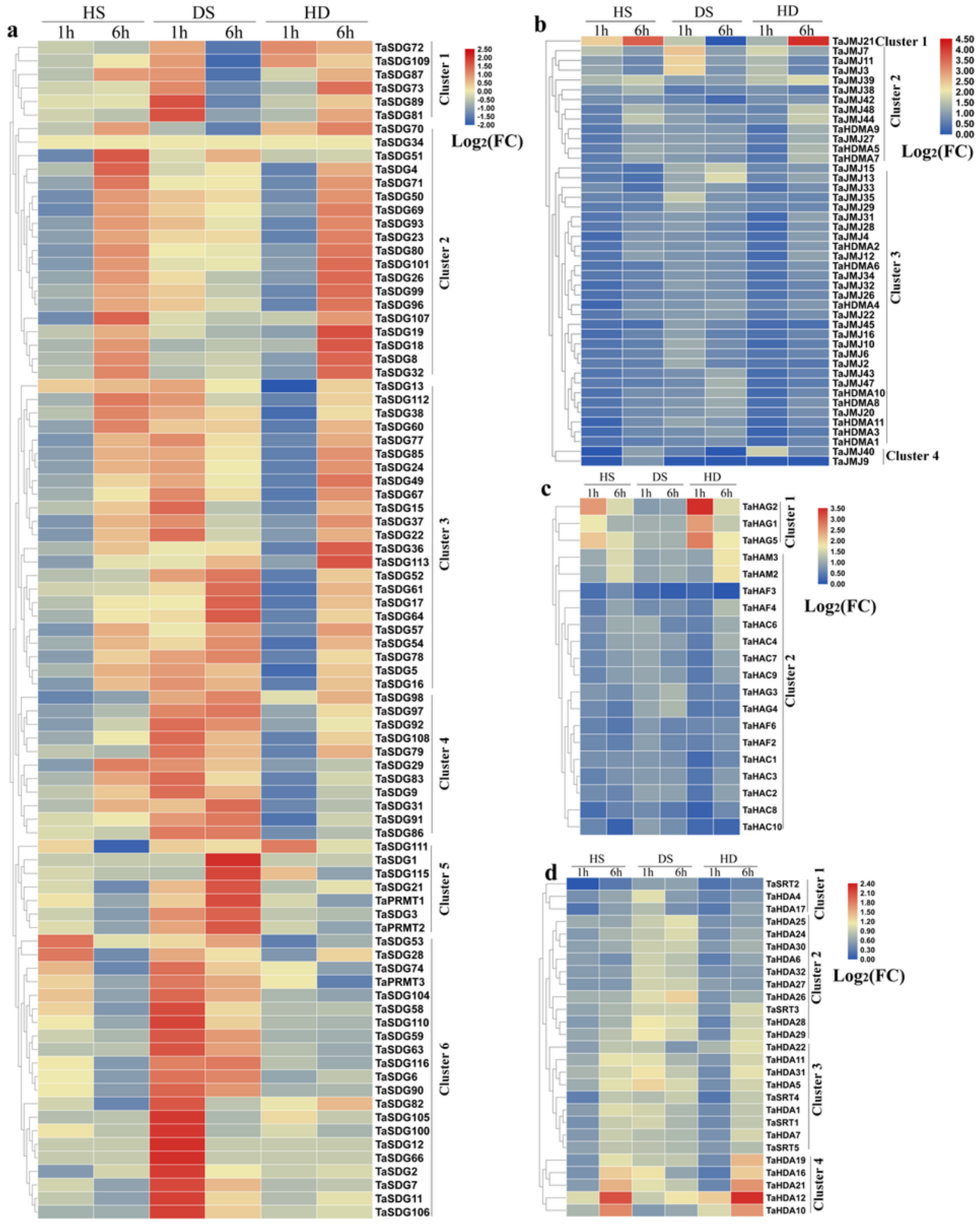

Figure 6

Expression pattern analysis of TaHMs in response to HS, DS and HD. a Differently expressed TaHMTs between control and HS, DS as well as HD group. b Differently expressed TaHDMs between control and HS, DS as well as HD group. c Differently expressed TaHATs between control and HS, DS as well as HD group. d Differently expressed TaHDACs between control and HS, DS as well as HD group. The log2FC data represent the level of upregulation or downregulation. FC: fold change. 

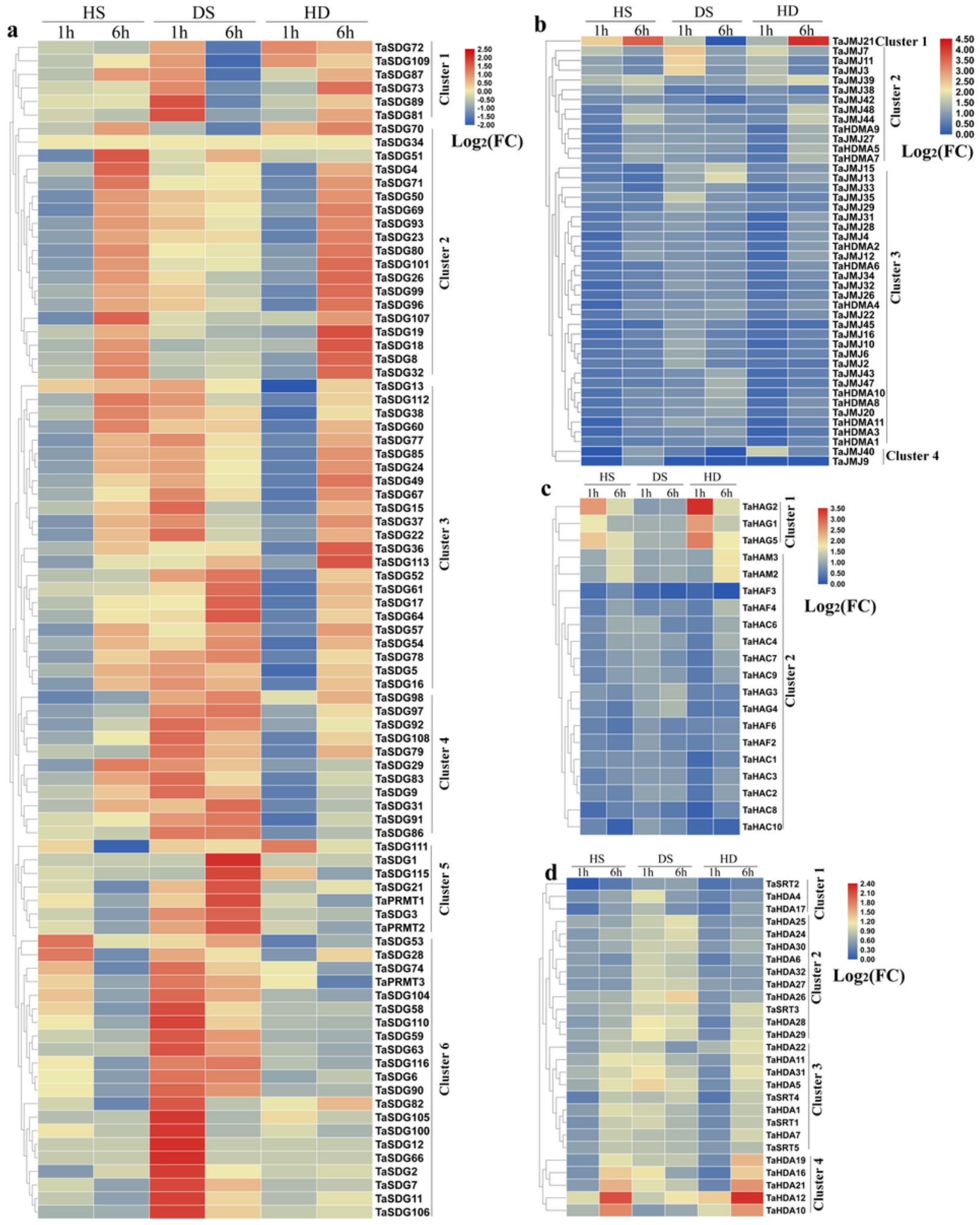

Figure 6

Expression pattern analysis of TaHMs in response to HS, DS and HD. a Differently expressed TaHMTs between control and HS, DS as well as HD group. b Differently expressed TaHDMs between control and HS, DS as well as HD group. c Differently expressed TaHATs between control and HS, DS as well as HD group. d Differently expressed TaHDACs between control and HS, DS as well as HD group. The log2FC data represent the level of upregulation or downregulation. FC: fold change. 


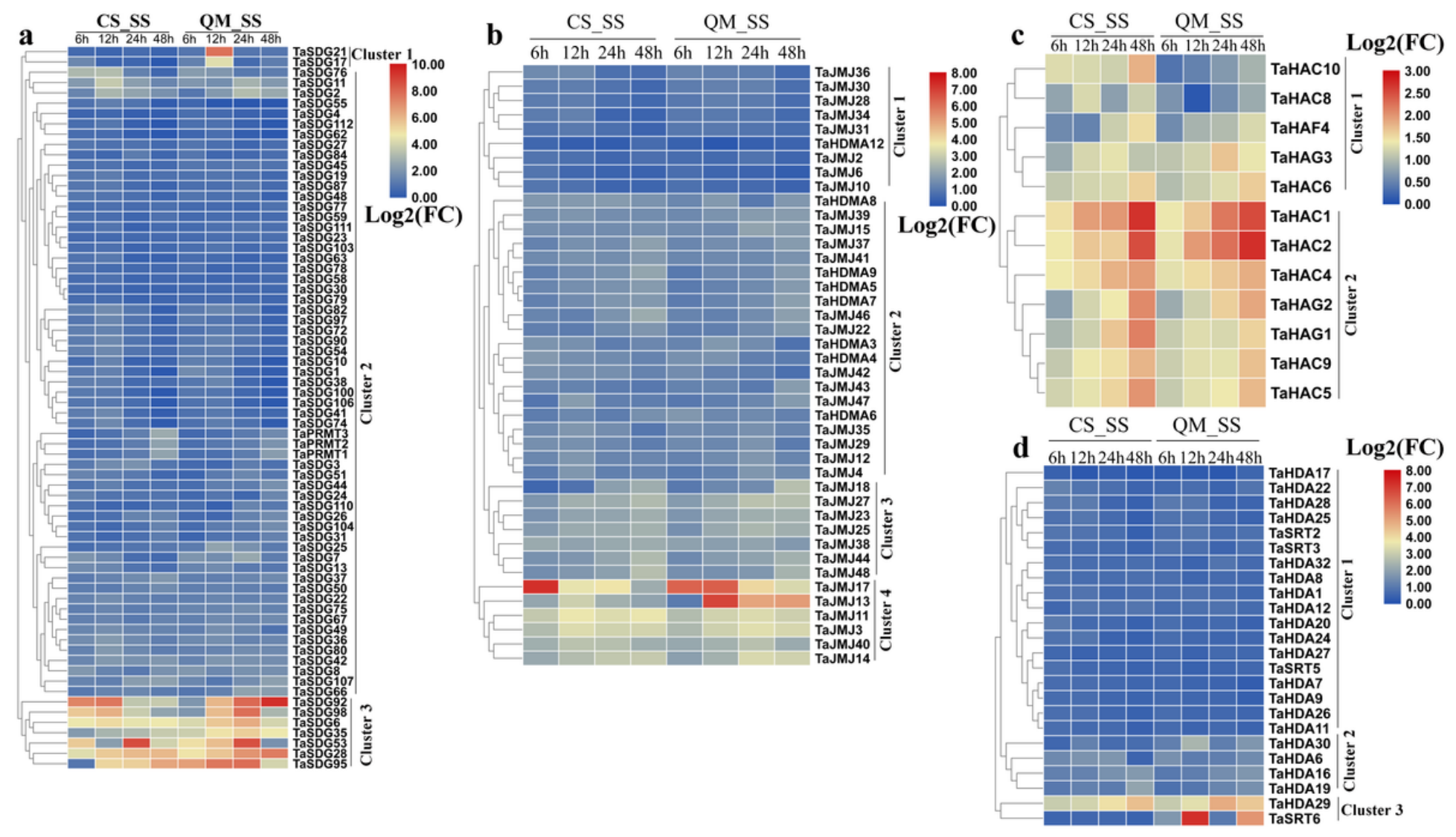

Figure 7

Expression pattern analysis of TaHMs in response to SS in two different varieties. a Differently expressed TaHMTs between control and SS group in CS and QM. b Differently expressed TaHDMs between control and SS group in CS and QM. c Differently expressed TaHATs between control and SS group in CS and QM. d Differently expressed TaHDACs between control and SS group in CS and QM. The log2FC data represent the level of upregulation or downregulation. FC: fold change. 


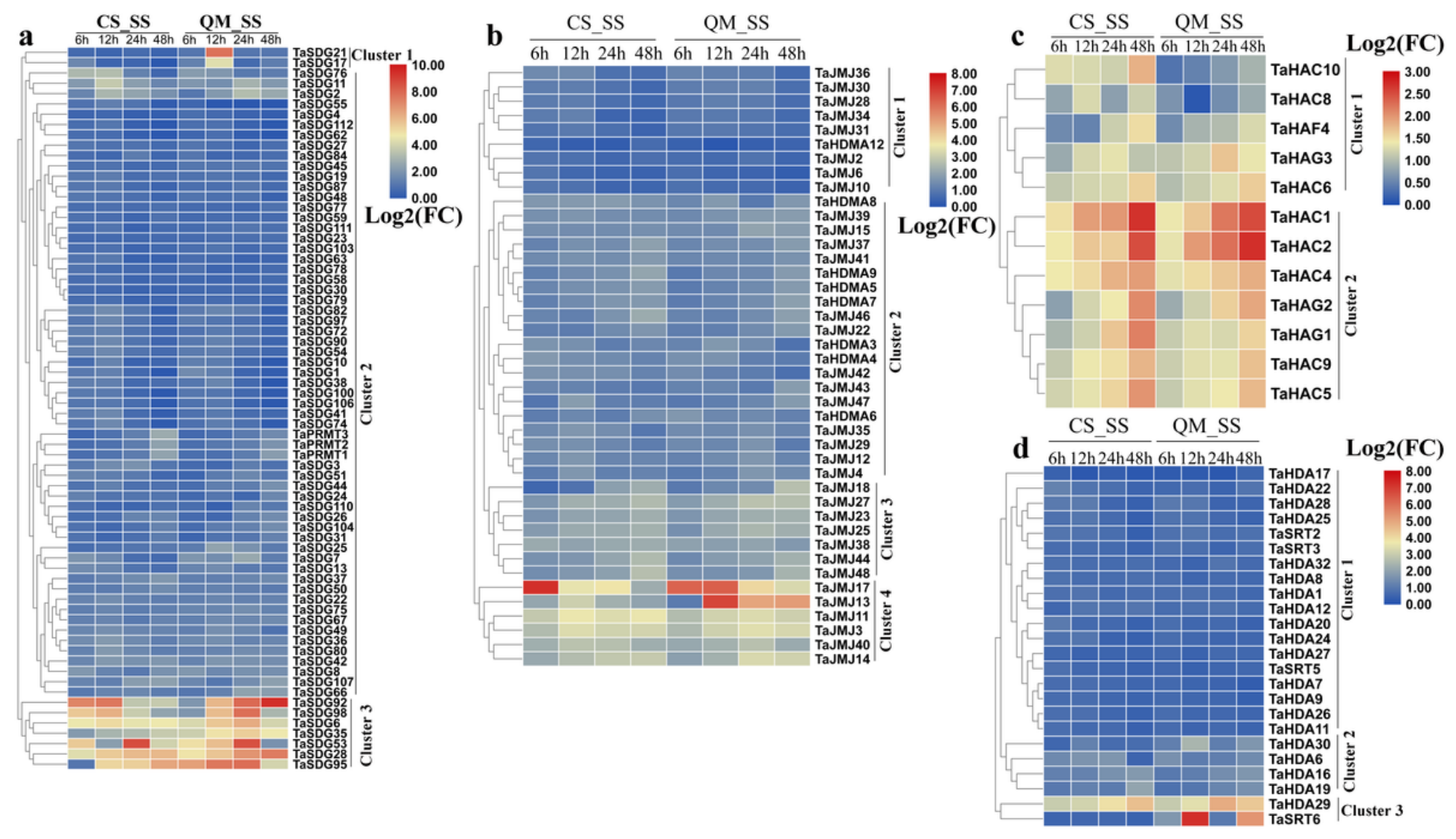

Figure 7

Expression pattern analysis of TaHMs in response to SS in two different varieties. a Differently expressed TaHMTs between control and SS group in CS and QM. b Differently expressed TaHDMs between control and SS group in CS and QM. c Differently expressed TaHATs between control and SS group in CS and QM. d Differently expressed TaHDACs between control and SS group in CS and QM. The log2FC data represent the level of upregulation or downregulation. FC: fold change. 


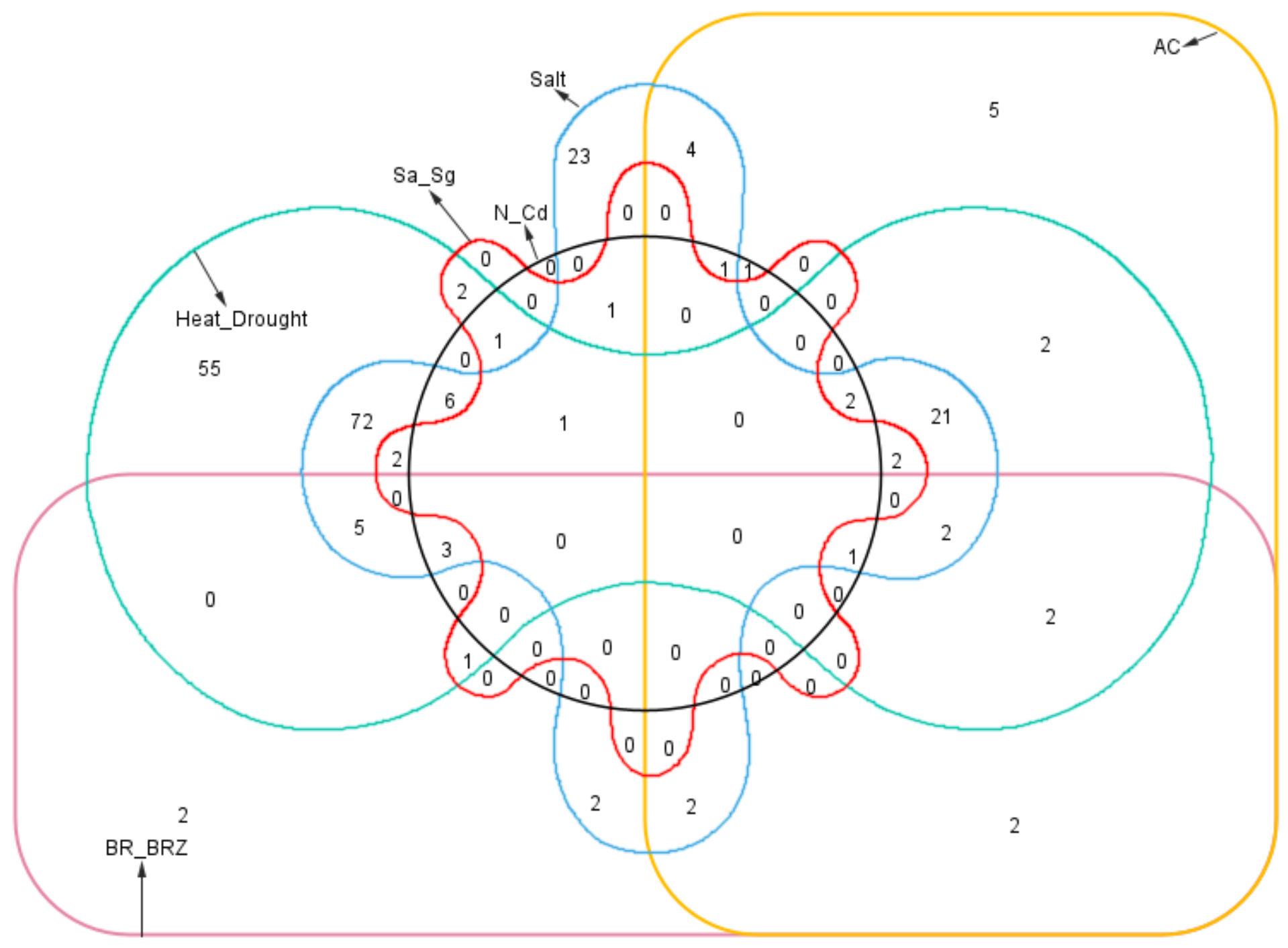

Figure 8

Venn analysis of DEGs under diverse growth and stress treatments. 


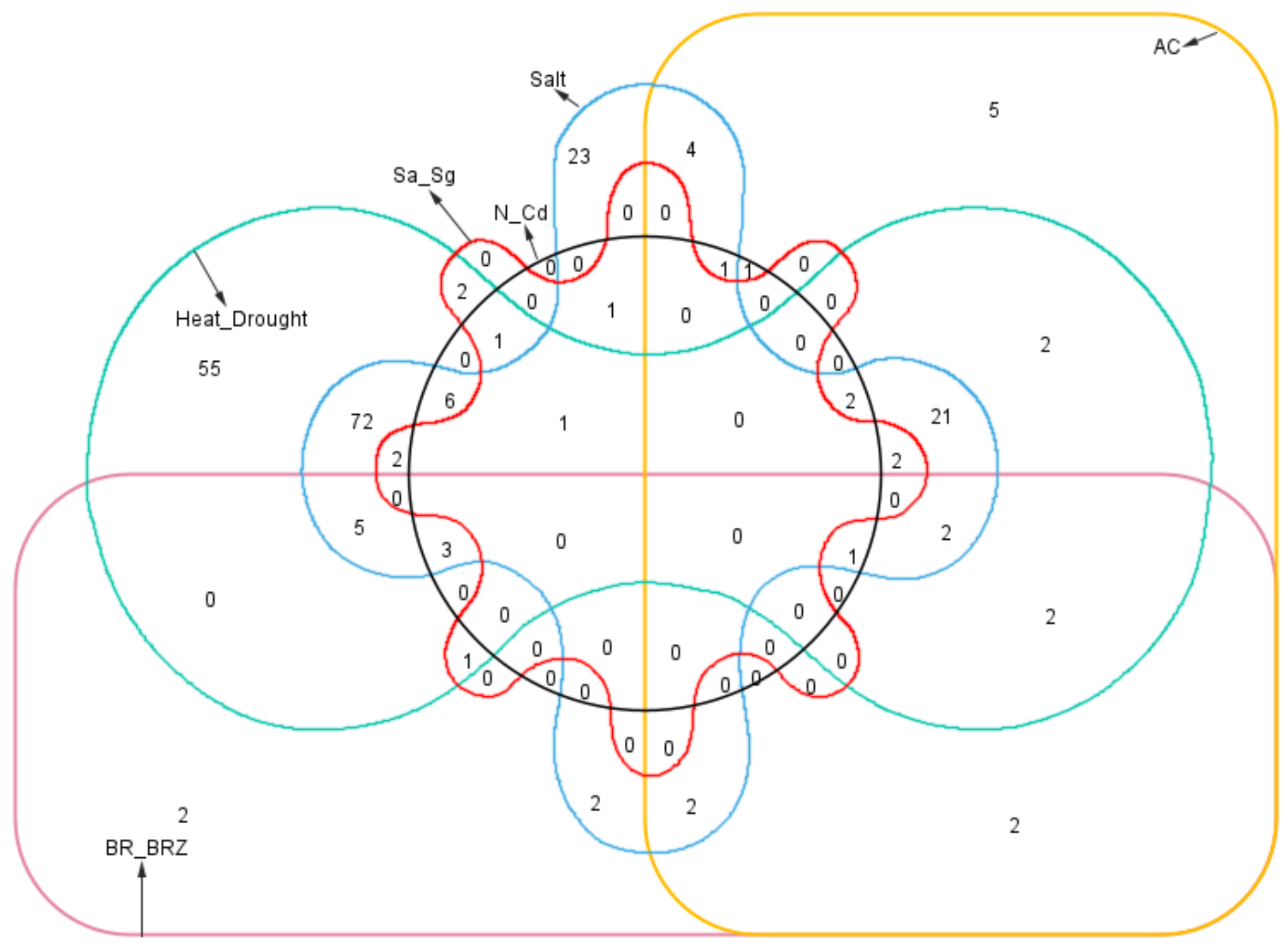

Figure 8

Venn analysis of DEGs under diverse growth and stress treatments. 

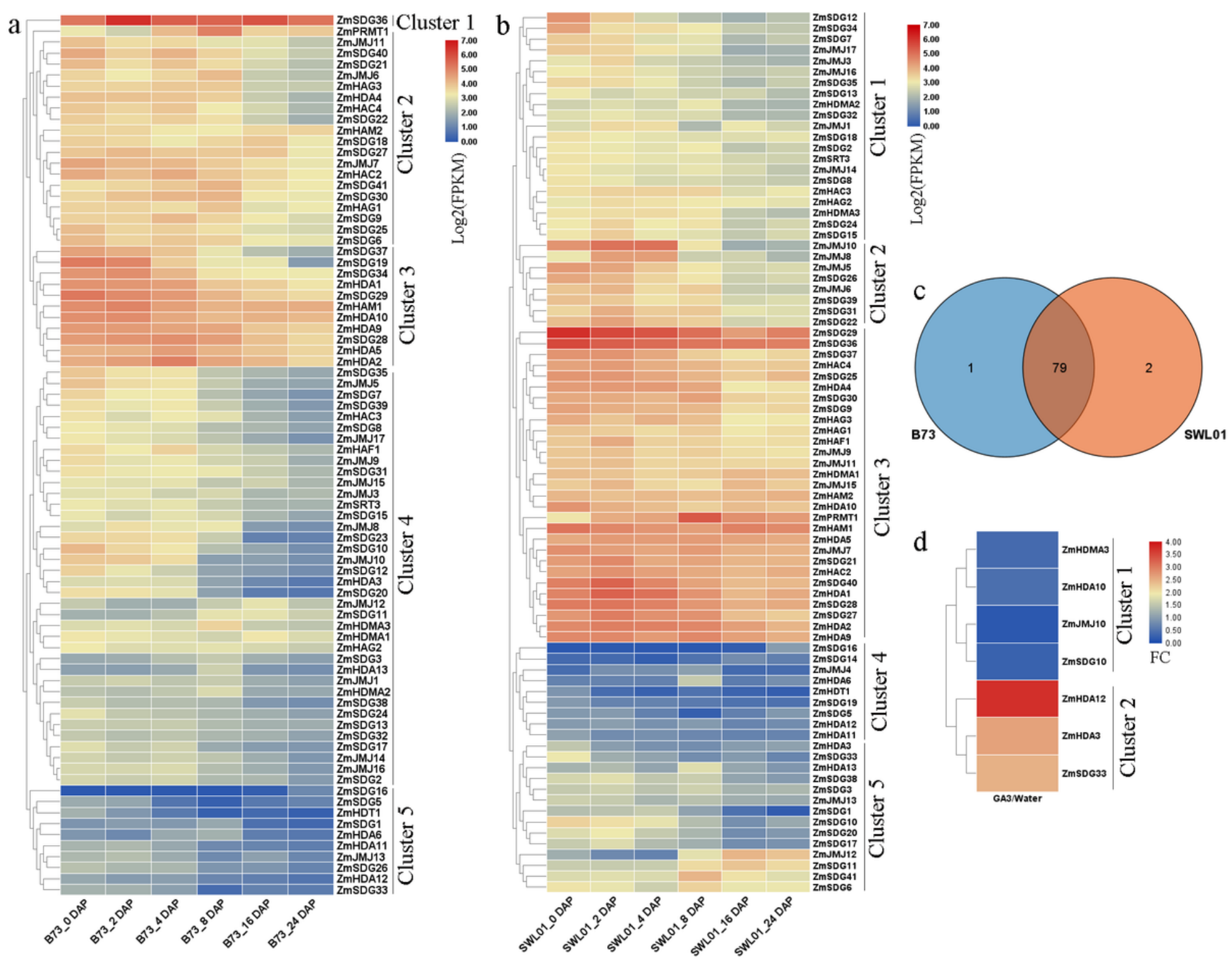

Figure 9

Expression profiles of ZmHMs genes in developing seeds and response to GA signal. a. Expression profiles of ZmHMs in developing B73 seeds. b. Expression profiles of ZmHMs in developing SWL01 seeds. c. Venn analysis of genes expressed in B73 and SWL01 seeds. d. Differently expressed TaHMs between GA-treated and control group. FPKM: Fragments Per Kilobase per Million. FC: fold change. 

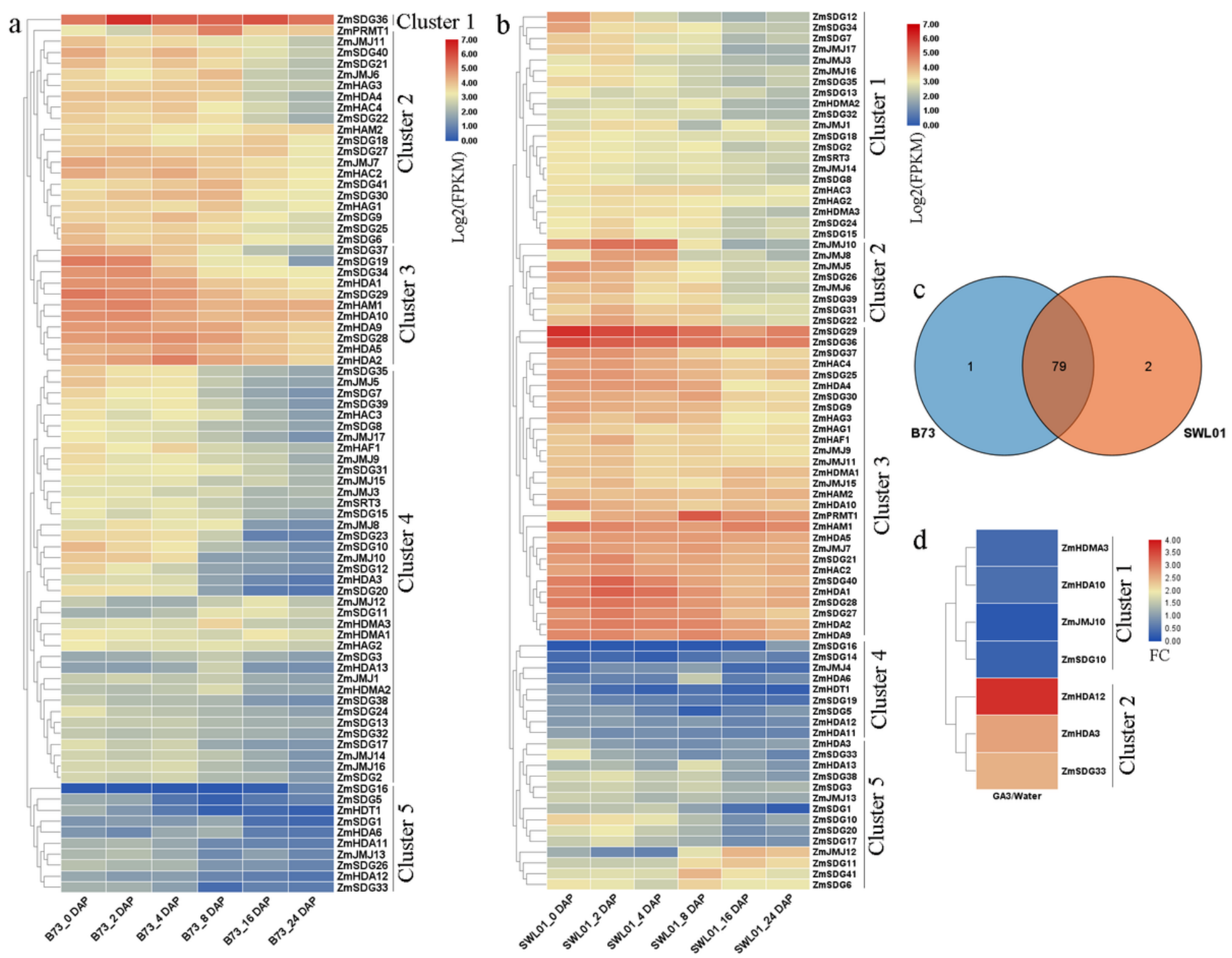

Figure 9

Expression profiles of $\mathrm{ZmHMs}$ genes in developing seeds and response to GA signal. a. Expression profiles of ZmHMs in developing B73 seeds. b. Expression profiles of ZmHMs in developing SWL01 seeds. c. Venn analysis of genes expressed in B73 and SWL01 seeds. d. Differently expressed TaHMs between GA-treated and control group. FPKM: Fragments Per Kilobase per Million. FC: fold change.

\section{Supplementary Files}

This is a list of supplementary files associated with this preprint. Click to download.

- Fig.S1.docx

- Fig.S1.docx

- Fig.S2.docx 
- Fig.S2.docx

- Fig.S3.docx

- Fig.S3.docx

- Fig.S4.docx

- Fig.S4.docx

- Fig.S5.docx

- Fig.S5.docx

- Fig.S6.docx

- Fig.S6.docx

- Fig.S7.docx

- Fig.S7.docx

- Fig.S8.docx

- Fig.S8.docx

- SupplementaryTables.xIsx

- SupplementaryTables.xlsx 